\title{
The General Relativistic Two Body Problem and the Effective One Body Formalism
}

\author{
Thibault Damour ${ }^{1}$ \\ ${ }^{1}$ Institut des Hautes Études Scientifiques, 35 route de \\ Chartres, 91440 Bures-sur-Yvette, France \\ E-mail: damour@ihes.fr
}

\begin{abstract}
A new analytical approach to the motion and radiation of (comparable mass) binary systems has been introduced in 1999 under the name of Effective One Body (EOB) formalism. We review the basic elements of this formalism, and discuss some of its recent developments. Several recent comparisons between EOB predictions and Numerical Relativity (NR) simulations have shown the aptitude of the EOB formalism to provide accurate descriptions of the dynamics and radiation of various binary systems (comprising black holes or neutron stars) in regimes that are inaccessible to other analytical approaches (such as the last orbits and the merger of comparable mass black holes). In synergy with NR simulations, post-Newtonian (PN) theory and Gravitational Self-Force (GSF) computations, the EOB formalism is likely to provide an efficient way of computing the very many accurate template waveforms that are needed for Gravitational Wave (GW) data analysis purposes.
\end{abstract}

\section{Introduction}

The general relativistic $N$-body problem has been investigated from the early days of Einstein's gravitation theory (and even earlier, because it was already tackled by Johannes Droste within the framework of the 1913 Einstein-Grossmann "Entwurf" theory). Here, we shall focus on the general relativistic two-body problem. This problem has been the subject of many investigations within the post-Newtonian $(\mathrm{PN})$ formalism, 
since the pioneering works of Einstein (1915; when $m_{1} \ll m_{2}$ ), Lorentz and Droste (1917), Levi-Civita (1937) and Einstein, Infeld and Hoffmann (1938). [see, e.g., [46 for a review and references to the early literature.] For many years, the first post-Newtonian (1PN) approximation (i.e. the inclusion of the leading-order relativistic corrections, proportional to $(v / c)^{2}$ or $G M / c^{2} r$, to the Newtonian equations of motion) appeared as being accurate enough for applying Einstein's theory to known binary systems. The situation changed in the mid 1970's with the discovery of the Hulse-Taylor binary pulsar PSR $1913+16$. The need to compare the accurate observations of this system (by Taylor and collaborators) to the predictions of Einstein's theory motivated the development of improved relativistic theories of binary systems, applicable to strongly selfgravitating bodies, and including terms up to the $2.5 \mathrm{PN}$ approximation (i.e. $O\left[(v / c)^{5}\right]$ beyond Newton). [See [45] and references therein.] The situation has again changed recently with the development of interferometric gravitational wave (GW) detectors, and the prospect of detecting the GW's emitted during the last orbits and the coalescence of binary systems made of black holes or neutron stars. The latter prospect motivated the development (or improvement) of several different methods of computing the motion and radiation of binary systems.

First, this motivated pushing PN calculations of the dynamics of binary systems to the 3PN level [90, 30, 62, 24, 89, with inclusion of $3.5 \mathrm{PN}$ radiation-reaction terms [105, 93, 99. Second, this motivated the development of new, accurate GW generation formalisms, notably the Blanchet-Damour-Iyer (matched) "multipolar post-Minkowskian" formalism 21, 22, 55, 56, 23, 17 and the "direct integration of the relaxed Einstein's equations" formalism of Will and collaborators [119, 118, 104, which extended previous work by Epstein and Wagoner [78 and Thorne [116. These GW generation formalisms allowed one to compute emitted gravitational waveforms with an unprecedented $\mathrm{PN}$ accuracy 1 . After the $1 \mathrm{PN}$ correction to the waveform [78, 117, 22, there is a 1.5PN "tail" (i.e. hereditary) correction [23, 120, 34, then a "direct" 2PN term [28, 27, 119, followed by higher-order corrections [18, 33, 32, 25, 26, 19, 14, 92, 31. [See [20 for a detailed account and more references.] Parallely to these improved PN computations of the GW emission of comparable-mass systems (with $m_{1} \sim m_{2}$ ), other authors developed the analytical theory of the GW emission of extreme mass-ratio systems (with $m_{1} \ll m_{2}$ ): see Refs. [106, 110, 113, 114, and the review of Sasaki and Tagoshi [111.

1 For gravitational waveforms, one conventionally defines the PN accuracy as the fractional PN accuracy with respect to the leading-order, $O\left(c^{-5}\right)$, quadrupolar emission. E.g., a $1 \mathrm{PN}$-accurate waveform retains next-to-leading order terms, i.e. terms smaller than the quadrupolar waveform by a factor $O\left(c^{-2}\right)$. 
Some of the PN calculations of the dynamics, and/or GW emission, of comparable-mass systems have been recently (re)done (e.g. the $3 \mathrm{PN}$ dynamics [80]) by using a somewhat different formalism, dubbed "effective field theory" 85. Let us, however, note that most of the technical aspects of the effective-field-theory approach had already been introduced and used before. For instance: (i) Ref. [50] discussed the (Fokker) two-body effective action due to the exchange of a linear field (of $\operatorname{spin} s=0,1$ and 2) ; (ii) Ref. [51] explicitly discussed the representation (and computation) of the (nonlinear) effective two-body action in terms of Feynman-like diagrams (made of concatenated propagators and vertices); (iii) The appendix A of Ref. 52 discussed finite-size effects in terms of nonminimal worldline couplings in the effective action; (iv) The (quantum field theory) technique of dimensional regularization (together with a diagrammatic analysis of ultraviolet divergences) was crucially used to derive the $3 \mathrm{PN}$ dynamics in Refs. 62, 24, and 3PN radiation in Ref. [26; and (v) The exponential parametrization of the metric (which suppresses the leadingorder gravitational cubic vertex) had been introduced in Ref. 22 and then standardly used in many $\mathrm{PN}$ works. It is, however, possible that the more systematic (and automatized) implementation of such diagrammatic methods, together with the tapping of standard techniques for computing Feynman graphs (as exemplified in [80]) may allow one to be more efficient in computing higher-order processes, or, at least, to open new ways of understanding them (see, in this respect, Ref. 84]).

Separately from these purely analytical approaches to the motion and radiation of binary systems, which have been developed since the early days of Einstein's theory, Numerical Relativity (NR) simulations of Einstein's equations have relatively recently (2005) succeeded (after more than thirty years of developmental progress) to stably evolve binary systems made of comparable mass black holes [107, 44, 4, 35. This has led to an explosion of works exploring many different aspects of strong-field dynamics in General Relativity, such as spin effects, recoil, relaxation of the deformed horizon formed during the coalescence of two black holes to a stationary Kerr black hole, high-velocity encounters, etc.; see 108 for a review. In addition, recently developed codes now allow one to accurately study the orbital dynamics, and the coalescence of binary neutron stars. Much physics remains to be explored in these systems, especially during and after the merger of the neutron stars (which involves a much more complex physics than the pure-gravity merger of two black holes).

Recently, a new source of information on the general relativistic twobody problem has opened: gravitational self-force (GSF) theory. This approach goes one step beyond the test-particle approximation (already used by Einstein in 1915) by taking into account self-field effects that 
modify the leading-order geodetic motion of a small mass $m_{1}$ moving in the background geometry generated by a large mass $m_{2}$. After some ground work (notably by DeWitt and Brehme) in the 1960's, GSF theory has recently undergone rapid developments (mixing theoretical and numerical methods) and can now yield numerical results that yield access to new information on strong-field dynamics in the extreme mass-ratio limit $m_{1} \ll m_{2}$. See Ref. [5] for a review.

Each of the approaches to the two-body problem mentioned so far, PN theory, NR simulations and GSF theory, have their advantages and their drawbacks. It has become recently clear that the best way to meet the challenge of accurately computing the gravitational waveforms (depending on several continuous parameters) that are needed for a successful detection and data analysis of GW signals in the upcoming LIGO/Virgo/GEO/... network of GW detectors is to combine knowledge from all the available approximation methods: PN, NR and GSF. Several ways of doing so are a priori possible. For instance, one could try to directly combine $\mathrm{PN}$-computed waveforms (approximately valid for large enough separations, say $\left.r \gtrsim 10 G\left(m_{1}+m_{2}\right) / c^{2}\right)$ with $\mathrm{NR}$ waveforms (computed with initial separations $r_{0}>10 G\left(m_{1}+m_{2}\right) / c^{2}$ and evolved up to merger and ringdown). However, this method still requires too much computational time, and is likely to lead to waveforms of rather poor accuracy, see, e.g., [88, 87, 97].

On the other hand, five years before NR succeeded in simulating the late inspiral and the coalescence of binary black holes, a new approach to the two-body problem was proposed: the Effective One Body (EOB) formalism [40, 41, 61, 47. The basic aim of the EOB formalism is to provide an analytical description of both the motion and the radiation of coalescing binary systems over the entire merger process, from the early inspiral, right through the plunge, merger and final ringdown. As early as 2000 [4] this method made several quantitative and qualitative predictions concerning the dynamics of the coalescence, and the corresponding GW radiation, notably: (i) a blurred transition from inspiral to a 'plunge' that is just a smooth continuation of the inspiral, (ii) a sharp transition, around the merger of the black holes, between a continued inspiral and a ring-down signal, and (iii) estimates of the radiated energy and of the spin of the final black hole. In addition, the effects of the individual spins of the black holes were investigated within the EOB [47, 38] and were shown to lead to a larger energy release for spins parallel to the orbital angular momentum, and to a dimensionless rotation parameter $J / E^{2}$ always smaller than unity at the end of the inspiral (so that a Kerr black hole can form right after the inspiral phase). All those predictions have been broadly confirmed by the results of the recent numerical simulations performed 
by several independent groups (for a review of numerical relativity results and references see [108]). Note that, in spite of the high computer power used in NR simulations, the calculation, checking and processing of one sufficiently long waveform (corresponding to specific values of the many continuous parameters describing the two arbitrary masses, the initial spin vectors, and other initial data) takes on the order of one month. This is a very strong argument for developing analytical models of waveforms.

\section{EOB description of the conservative dynamics of two body systems}

Before reviewing some of the technical aspects of the EOB method, let us indicate the historical roots of this method. First, we note that the EOB approach comprises three, rather separate, ingredients:

(i) a description of the conservative (Hamiltonian) part of the dynamics of two bodies;

(ii) an expression for the radiation-reaction part of the dynamics;

(iii) a description of the GW waveform emitted by a coalescing binary system.

For each one of these ingredients, the essential inputs that are used in EOB works are high-order post-Newtonian (PN) expanded results which have been obtained by many years of work, by many researchers (see references above). However, one of the key ideas in the EOB philosophy is to avoid using PN results in their original "Taylor-expanded" form (i.e. $\left.c_{0}+c_{1} v / c+c_{2} v^{2} / c^{2}+c_{3} v^{3} / c^{3}+\cdots+c_{n} v^{n} / c^{n}\right)$, but to use them instead in some resummed form (i.e. some non-polynomial function of $v / c$, defined so as to incorporate some of the expected non-perturbative features of the exact result). The basic ideas and techniques for resumming each ingredient of the EOB are different and have different historical roots.

Concerning the first ingredient, i.e. the EOB Hamiltonian, it was inspired by an approach to electromagnetically interacting quantum twobody systems introduced by Brézin, Itzykson and Zinn-Justin [36.

The resummation of the second ingredient, i.e. the EOB radiationreaction force $\mathcal{F}$, was initially inspired by the Padé resummation of the flux function introduced by Damour, Iyer and Sathyaprakash [58. More recently, a new and more sophisticated resummation technique for the radiation reaction force $\mathcal{F}$ has been introduced by Damour and Nagar [57, 67].

As for the third ingredient, i.e. the EOB description of the waveform emitted by a coalescing black hole binary, it was mainly inspired by the 
work of Davis, Ruffini and Tiomno [77] which discovered the transition between the plunge signal and a ringing tail when a particle falls into a black hole. [Additional motivation for the EOB treatment of the transition from plunge to ring-down came from work on the, so-called, "close limit approximation" [109.] In addition, a very efficient resummation of the waveform has been introduced by Damour, Iyer and Nagar [64, 66, 57. It will be discussed in detail below.

Within the usual PN formalism, the conservative dynamics of a twobody system is currently fully known up to the 3PN level 90, 30, 62, 24, 89, 80, (see below for the partial knowledge beyond the 3PN level). Going to the center of mass of the system $\left(\boldsymbol{p}_{1}+\boldsymbol{p}_{2}=0\right)$, the $3 \mathrm{PN}$-accurate Hamiltonian (in Arnowitt-Deser-Misner-type coordinates) describing the relative motion, $\boldsymbol{q}=\boldsymbol{q}_{1}-\boldsymbol{q}_{2}, \boldsymbol{p}=\boldsymbol{p}_{1}=-\boldsymbol{p}_{2}$, has the structure

$$
H_{3 \mathrm{PN}}^{\text {relative }}(\boldsymbol{q}, \boldsymbol{p})=H_{0}(\boldsymbol{q}, \boldsymbol{p})+\frac{1}{c^{2}} H_{2}(\boldsymbol{q}, \boldsymbol{p})+\frac{1}{c^{4}} H_{4}(\boldsymbol{q}, \boldsymbol{p})+\frac{1}{c^{6}} H_{6}(\boldsymbol{q}, \boldsymbol{p}),
$$

where

$$
H_{0}(\boldsymbol{q}, \boldsymbol{p})=\frac{1}{2 \mu} \boldsymbol{p}^{2}-\frac{G M \mu}{|\boldsymbol{q}|}
$$

with

$$
M \equiv m_{1}+m_{2} \quad \text { and } \quad \mu \equiv m_{1} m_{2} / M,
$$

corresponds to the Newtonian approximation to the relative motion, while $H_{2}$ describes $1 \mathrm{PN}$ corrections, $H_{4} 2 \mathrm{PN}$ ones and $H_{6} 3 \mathrm{PN}$ ones. In terms of the rescaled variables $\boldsymbol{q}^{\prime} \equiv \boldsymbol{q} / G M, \boldsymbol{p}^{\prime} \equiv \boldsymbol{p} / \mu$, the explicit form (after dropping the primes for readability) of the $3 \mathrm{PN}$-accurate rescaled Hamiltonian $\widehat{H} \equiv H / \mu$ reads 60, 65, 62.

$$
\begin{gathered}
\widehat{H}_{N}(\boldsymbol{q}, \boldsymbol{p})=\frac{\boldsymbol{p}^{2}}{2}-\frac{1}{q} \\
\widehat{H}_{1 \mathrm{PN}}(\boldsymbol{q}, \boldsymbol{p})=\frac{1}{8}(3 \nu-1)\left(\boldsymbol{p}^{2}\right)^{2}-\frac{1}{2}\left[(3+\nu) \boldsymbol{p}^{2}+\nu(\boldsymbol{n} \cdot \boldsymbol{p})^{2}\right] \frac{1}{q}+\frac{1}{2 q^{2}} \\
\widehat{H}_{2 \mathrm{PN}}(\boldsymbol{q}, \boldsymbol{p})=\frac{1}{16}\left(1-5 \nu+5 \nu^{2}\right)\left(\boldsymbol{p}^{2}\right)^{3} \\
+\frac{1}{8}\left[\left(5-20 \nu-3 \nu^{2}\right)\left(\boldsymbol{p}^{2}\right)^{2}-2 \nu^{2}(\boldsymbol{n} \cdot \boldsymbol{p})^{2} \boldsymbol{p}^{2}-3 \nu^{2}(\boldsymbol{n} \cdot \boldsymbol{p})^{4}\right] \frac{1}{q} \\
+\frac{1}{2}\left[(5+8 \nu) \boldsymbol{p}^{2}+3 \nu(\boldsymbol{n} \cdot \boldsymbol{p})^{2}\right] \frac{1}{q^{2}}-\frac{1}{4}(1+3 \nu) \frac{1}{q^{3}} \\
\widehat{H}_{3 \mathrm{PN}}(\boldsymbol{q}, \boldsymbol{p})=\frac{1}{128}\left(-5+35 \nu-70 \nu^{2}+35 \nu^{3}\right)\left(\boldsymbol{p}^{2}\right)^{4}
\end{gathered}
$$




$$
\begin{aligned}
+\frac{1}{16}[(-7+ & \left.42 \nu-53 \nu^{2}-5 \nu^{3}\right)\left(\boldsymbol{p}^{2}\right)^{3}+(2-3 \nu) \nu^{2}(\boldsymbol{n} \cdot \boldsymbol{p})^{2}\left(\boldsymbol{p}^{2}\right)^{2} \\
& \left.+3(1-\nu) \nu^{2}(\boldsymbol{n} \cdot \boldsymbol{p})^{4} \boldsymbol{p}^{2}-5 \nu^{3}(\boldsymbol{n} \cdot \boldsymbol{p})^{6}\right] \frac{1}{q} \\
+\left[\frac{1}{16}\left(-27+136 \nu+109 \nu^{2}\right)\left(\boldsymbol{p}^{2}\right)^{2}+\frac{1}{16}(17+30 \nu) \nu(\boldsymbol{n} \cdot \boldsymbol{p})^{2} \boldsymbol{p}^{2}\right. & \\
& \left.+\frac{1}{12}(5+43 \nu) \nu(\boldsymbol{n} \cdot \boldsymbol{p})^{4}\right] \frac{1}{q^{2}} \\
+ & \left\{\left[-\frac{25}{8}+\left(\frac{1}{64} \pi^{2}-\frac{335}{48}\right) \nu-\frac{23}{8} \nu^{2}\right] \boldsymbol{p}^{2}\right. \\
+ & \left.\left(-\frac{85}{16}-\frac{3}{64} \pi^{2}-\frac{7}{4} \nu\right) \nu(\boldsymbol{n} \cdot \boldsymbol{p})^{2}\right\} \frac{1}{q^{3}} \\
& +\left[\frac{1}{8}+\left(\frac{109}{12}-\frac{21}{32} \pi^{2}\right) \nu\right] \frac{1}{q^{4}} .
\end{aligned}
$$

In these formulas $\nu$ denotes the symmetric mass ratio:

$$
\nu \equiv \frac{\mu}{M} \equiv \frac{m_{1} m_{2}}{\left(m_{1}+m_{2}\right)^{2}} .
$$

The dimensionless parameter $\nu$ varies between 0 (extreme mass ratio case) and $\frac{1}{4}$ (equal mass case) and plays the rôle of a deformation parameter away from the test-mass limit.

It is well known that, at the Newtonian approximation, $H_{0}(\boldsymbol{q}, \boldsymbol{p})$ can be thought of as describing a 'test particle' of mass $\mu$ orbiting around an 'external mass' GM. The EOB approach is a general relativistic generalization of this fact. It consists in looking for an 'effective external spacetime geometry' $g_{\mu \nu}^{\text {eff }}\left(x^{\lambda} ; G M, \nu\right)$ such that the geodesic dynamics of a 'test particle' of mass $\mu$ within $g_{\mu \nu}^{\text {eff }}\left(x^{\lambda}, G M, \nu\right)$ is equivalent (when expanded in powers of $1 / c^{2}$ ) to the original, relative PN-expanded dynamics (11).

Let us explain the idea, proposed in [40, for establishing a 'dictionary' between the real relative-motion dynamics, (1), and the dynamics of an 'effective' particle of mass $\mu$ moving in $g_{\mu \nu}^{\text {eff }}\left(x^{\lambda}, G M, \nu\right)$. The idea consists in 'thinking quantum mechanically. Instead of thinking in terms of a classical Hamiltonian, $H(\boldsymbol{q}, \boldsymbol{p})$ (such as $H_{3 \mathrm{PN}}^{\text {relative }}$, Eq. (1)), and of its classical bound orbits, we can think in terms of the quantized

2 This is related to an idea emphasized many times by John Archibald Wheeler: quantum mechanics can often help us in going to the essence of classical mechanics. 
energy levels $E(n, \ell)$ of the quantum bound states of the Hamiltonian operator $H(\hat{\boldsymbol{q}}, \hat{\boldsymbol{p}})$. These energy levels will depend on two (integer valued) quantum numbers $n$ and $\ell$. Here (for a spherically symmetric interaction, as appropriate to $\left.H^{\text {relative }}\right)$, $\ell$ parametrizes the total orbital angular momentum $\left(\boldsymbol{L}^{2}=\ell(\ell+1) \hbar^{2}\right)$, while $n$ represents the 'principal quantum number' $n=\ell+n_{r}+1$, where $n_{r}$ (the 'radial quantum number') denotes the number of nodes in the radial wave function. The third 'magnetic quantum number' $m$ (with $-\ell \leq m \leq \ell$ ) does not enter the energy levels because of the spherical symmetry of the two-body interaction (in the center of mass frame). For instance, the non-relativistic Newton interaction Eq. (2) gives rise to the well-known result

$$
E_{0}(n, \ell)=-\frac{1}{2} \mu\left(\frac{G M \mu}{n \hbar}\right)^{2},
$$

which depends only on $n$ (this is the famous Coulomb degeneracy). When considering the PN corrections to $H_{0}$, as in Eq. (11), one gets a more complicated expression of the form

$$
\begin{gathered}
E_{3 \mathrm{PN}}^{\mathrm{relative}}(n, \ell)=-\frac{1}{2} \mu \frac{\alpha^{2}}{n^{2}}\left[1+\frac{\alpha^{2}}{c^{2}}\left(\frac{c_{11}}{n \ell}+\frac{c_{20}}{n^{2}}\right)\right. \\
\left.+\frac{\alpha^{4}}{c^{4}}\left(\frac{c_{13}}{n \ell^{3}}+\frac{c_{22}}{n^{2} \ell^{2}}+\frac{c_{31}}{n^{3} \ell}+\frac{c_{40}}{n^{4}}\right)+\frac{\alpha^{6}}{c^{6}}\left(\frac{c_{15}}{n \ell^{5}}+\ldots+\frac{c_{60}}{n^{6}}\right)\right],
\end{gathered}
$$

where we have set $\alpha \equiv G M \mu / \hbar=G m_{1} m_{2} / \hbar$, and where we consider, for simplicity, the (quasi-classical) limit where $n$ and $\ell$ are large numbers. The 2PN-accurate version of Eq. (10) had been derived by Damour and Schäfer [76] as early as 1988 while its 3PN-accurate version was derived by Damour, Jaranowski and Schäfer in 1999 60. The dimensionless coefficients $c_{p q}$ are functions of the symmetric mass ratio $\nu \equiv \mu / M$, for instance $c_{40}=\frac{1}{8}\left(145-15 \nu+\nu^{2}\right)$. In classical mechanics (i.e. for large $n$ and $\ell$ ), it is called the 'Delaunay Hamiltonian', i.e. the Hamiltonian expressed in terms of the action variables $J=\ell \hbar=\frac{1}{2 \pi} \oint p_{\varphi} d \varphi$, and $N=n \hbar=I_{r}+J$, with $I_{r}=\frac{1}{2 \pi} \oint p_{r} d r$.

The energy levels (10) encode, in a gauge-invariant way, the 3PNaccurate relative dynamics of a 'real' binary. Let us now consider an auxiliary problem: the 'effective' dynamics of one body, of mass $\mu$, following (modulo the $Q$ term discussed below) a geodesic in some $\nu$ dependent 'effective external' (spherically symmetric) metri 4

$$
g_{\mu \nu}^{\mathrm{eff}} d x^{\mu} d x^{\nu}=-A(R ; \nu) c^{2} d T^{2}+B(R ; \nu) d R^{2}+R^{2}\left(d \theta^{2}+\sin ^{2} \theta d \varphi^{2}\right) .
$$

3 We consider, for simplicity, 'equatorial' motions with $m=\ell$, i.e., classically, $\theta=\frac{\pi}{2}$.

${ }^{4}$ It is convenient to write the 'effective metric' in Schwarzschild-like coordinates. Note that the effective radial coordinate $R$ differs from the two-body ADM- 
Here, the a priori unknown metric functions $A(R ; \nu)$ and $B(R ; \nu)$ will be constructed in the form of expansions in $G M / c^{2} R$ :

$$
\begin{aligned}
& A(R ; \nu)=1+\widetilde{a}_{1} \frac{G M}{c^{2} R}+\widetilde{a}_{2}\left(\frac{G M}{c^{2} R}\right)^{2}+\widetilde{a}_{3}\left(\frac{G M}{c^{2} R}\right)^{3}+\widetilde{a}_{4}\left(\frac{G M}{c^{2} R}\right)^{4}+\cdots ; \\
& B(R ; \nu)=1+\widetilde{b}_{1} \frac{G M}{c^{2} R}+\widetilde{b}_{2}\left(\frac{G M}{c^{2} R}\right)^{2}+b_{3}\left(\frac{G M}{c^{2} R}\right)^{3}+\cdots,
\end{aligned}
$$

where the dimensionless coefficients $\widetilde{a}_{n}, \widetilde{b}_{n}$ depend on $\nu$. From the Newtonian limit, it is clear that we should set $\widetilde{a}_{1}=-2$. In addition, as $\nu$ can be viewed as a deformation parameter away from the test-mass limit, we require that the effective metric (11) tend to the Schwarzschild metric (of mass $M$ ) as $\nu \rightarrow 0$, i.e. that

$$
A(R ; \nu=0)=1-2 G M / c^{2} R=B^{-1}(R ; \nu=0) .
$$

Let us now require that the dynamics of the "one body" $\mu$ within the effective metric $g_{\mu \nu}^{\text {eff }}$ be described by an "effective" mass-shell condition of the form

$$
g_{\text {eff }}^{\mu \nu} p_{\mu}^{\mathrm{eff}} p_{\nu}^{\mathrm{eff}}+\mu^{2} c^{2}+Q\left(p_{\mu}^{\mathrm{eff}}\right)=0,
$$

where $Q(p)$ is (at least) quartic in $p$. Then by solving (by separation of variables) the corresponding 'effective' Hamilton-Jacobi equation

$$
\begin{gathered}
g_{\mathrm{eff}}^{\mu \nu} \frac{\partial S_{\mathrm{eff}}}{\partial x^{\mu}} \frac{\partial S_{\mathrm{eff}}}{\partial x^{\nu}}+\mu^{2} c^{2}+Q\left(\frac{\partial S}{\partial x^{\mu}}\right)=0, \\
S_{\mathrm{eff}}=-\mathcal{E}_{\mathrm{eff}} t+J_{\mathrm{eff}} \varphi+S_{\mathrm{eff}}(R),
\end{gathered}
$$

one can straightforwardly compute (in the quasi-classical, large quantum numbers limit) the effective Delaunay Hamiltonian $\mathcal{E}_{\text {eff }}\left(N_{\text {eff }}, J_{\text {eff }}\right)$, with $N_{\text {eff }}=n_{\text {eff }} \hbar, J_{\text {eff }}=\ell_{\text {eff }} \hbar$ (where $N_{\text {eff }}=J_{\text {eff }}+I_{R}^{\text {eff }}$, with $I_{R}^{\text {eff }}=\frac{1}{2 \pi} \oint p_{R}^{\text {eff }} d R$, $\left.P_{R}^{\text {eff }}=\partial S_{\text {eff }}(R) / d R\right)$. This yields a result of the form

$$
\begin{aligned}
\mathcal{E}_{\mathrm{eff}}\left(n_{\mathrm{eff}}, \ell_{\mathrm{eff}}\right) & =\mu c^{2}-\frac{1}{2} \mu \frac{\alpha^{2}}{n_{\mathrm{eff}}^{2}}\left[1+\frac{\alpha^{2}}{c^{2}}\left(\frac{c_{11}^{\mathrm{eff}}}{n_{\mathrm{eff}} \ell_{\mathrm{eff}}}+\frac{c_{20}^{\mathrm{eff}}}{n_{\mathrm{eff}}^{2}}\right)\right. \\
& +\frac{\alpha^{4}}{c^{4}}\left(\frac{c_{13}^{\mathrm{eff}}}{n_{\mathrm{eff}} \ell_{\mathrm{eff}}^{3}}+\frac{c_{22}^{\mathrm{eff}}}{n_{\mathrm{eff}}^{2} \ell_{\mathrm{eff}}^{2}}+\frac{c_{31}^{\mathrm{eff}}}{n_{\mathrm{eff}}^{3} \ell_{\mathrm{eff}}}+\frac{c_{40}^{\mathrm{eff}}}{n_{\mathrm{eff}}^{4}}\right) \\
& \left.+\frac{\alpha^{6}}{c^{6}}\left(\frac{c_{15}^{\mathrm{eff}}}{n_{\mathrm{eff}} \ell_{\mathrm{eff}}^{5}}+\ldots+\frac{c_{60}^{\mathrm{eff}}}{n_{\mathrm{eff}}^{6}}\right)\right],
\end{aligned}
$$

coordinate relative distance $R^{\mathrm{ADM}}=|\boldsymbol{q}|$. The transformation between the two coordinate systems has been determined in Refs. [40, 61. 
where the dimensionless coefficients $c_{p q}^{\text {eff }}$ are now functions of the unknown coefficients $\widetilde{a}_{n}, \widetilde{b}_{n}$ entering the looked for 'external' metric coefficients (12).

At this stage, one needs to define a 'dictionary' between the real (relative) two-body dynamics, summarized in Eq. (10), and the effective one-body one, summarized in Eq. (14). As, on both sides, quantum mechanics tells us that the action variables are quantized in integers $\left(N_{\text {real }}=n \hbar, N_{\text {eff }}=n_{\text {eff }} \hbar\right.$, etc. $)$ it is most natural to identify $n=n_{\text {eff }}$ and $\ell=\ell_{\text {eff }}$. One then still needs a rule for relating the two different energies $E_{\text {real }}^{\text {relative }}$ and $\mathcal{E}_{\text {eff. }}$. Ref. [40] proposed to look for a general map between the real energy levels and the effective ones (which, as seen when comparing (10) and (14), cannot be directly identified because they do not include the same rest-mass contribution 5), namely

$$
\begin{aligned}
& \frac{\mathcal{E}_{\text {eff }}}{\mu c^{2}}-1=f\left(\frac{E_{\text {real }}^{\text {relative }}}{\mu c^{2}}\right)=\frac{E_{\text {real }}^{\text {relative }}}{\mu c^{2}}\left(1+\alpha_{1} \frac{E_{\text {real }}^{\text {relative }}}{\mu c^{2}}+\alpha_{2}\left(\frac{E_{\text {real }}^{\text {relative }}}{\mu c^{2}}\right)^{2}\right. \\
& \left.+\alpha_{3}\left(\frac{E_{\text {real }}^{\text {relative }}}{\mu c^{2}}\right)^{3}+\ldots\right) .
\end{aligned}
$$

The 'correspondence' between the real and effective energy levels is illustrated in Fig. 1]

Finally, identifying $\mathcal{E}_{\text {eff }}(n, \ell) / \mu c^{2}$ to $1+f\left(E_{\text {real }}^{\text {relative }}(n, \ell) / \mu c^{2}\right)$ yields a system of equations for determining the unknown EOB coefficients $\widetilde{a}_{n}, \widetilde{b}_{n}, \alpha_{n}$, as well as the three coefficients $z_{1}, z_{2}, z_{3}$ parametrizing a general 3PN-level quartic mass-shell deformation:

$$
Q_{3 \mathrm{PN}}(p)=\frac{1}{c^{6}} \frac{1}{\mu^{2}}\left(\frac{G M}{R}\right)^{2}\left[z_{1} \boldsymbol{p}^{4}+z_{2} \boldsymbol{p}^{2}(\boldsymbol{n} \cdot \boldsymbol{p})^{2}+z_{3}(\boldsymbol{n} \cdot \boldsymbol{p})^{4}\right] .
$$

[The need for introducing a quartic mass-shell deformation $Q$ only arises at the $3 \mathrm{PN}$ level.]

The above system of equations for $\widetilde{a}_{n}, \widetilde{b}_{n}, \alpha_{n}$ (and $z_{i}$ at $3 \mathrm{PN}$ ) was studied at the $2 \mathrm{PN}$ level in Ref. [40, and at the $3 \mathrm{PN}$ level in Ref. 61. At the $2 \mathrm{PN}$ level it was found that, if one further imposes the natural condition $\widetilde{b}_{1}=+2$ (so that the linearized effective metric coincides with the linearized Schwarzschild metric with mass $M=m_{1}+m_{2}$ ), there exists a unique solution for the remaining five unknown coefficients $\widetilde{a}_{2}, \widetilde{a}_{3}, \widetilde{b}_{2}, \alpha_{1}$ and $\alpha_{2}$. This solution is very simple:

$$
\widetilde{a}_{2}=0, \quad \widetilde{a}_{3}=2 \nu, \quad \widetilde{b}_{2}=4-6 \nu, \quad \alpha_{1}=\frac{\nu}{2}, \quad \alpha_{2}=0 .
$$

${ }^{5}$ Indeed $E_{\text {real }}^{\text {total }}=M c^{2}+E_{\text {real }}^{\text {relative }}=M c^{2}+$ Newtonian terms $+1 \mathrm{PN} / c^{2}+\cdots$, while $\mathcal{E}_{\text {effective }}=\mu c^{2}+N+1 \mathrm{PN} / c^{2}+\cdots$. 


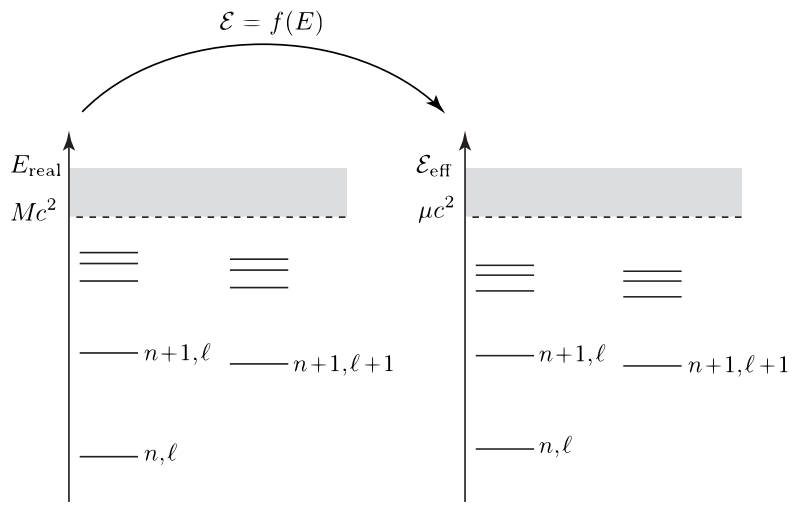

Figure 1. Sketch of the correspondence between the quantized energy levels of the real and effective conservative dynamics. $n$ denotes the 'principal quantum number' $\left(n=n_{r}+\ell+1\right.$, with $n_{r}=0,1, \ldots$ denoting the number of nodes in the radial function), while $\ell$ denotes the (relative) orbital angular momentum $\left(\boldsymbol{L}^{2}=\ell(\ell+1) \hbar^{2}\right)$. Though the EOB method is purely classical, it is conceptually useful to think in terms of the underlying (Bohr-Sommerfeld) quantization conditions of the action variables $I_{R}$ and $J$ to motivate the identification between $n$ and $\ell$ in the two dynamics.

At the $3 \mathrm{PN}$ level, it was found that the system of equations is consistent, and underdetermined in that the general solution can be parametrized by the arbitrary values of $z_{1}$ and $z_{2}$. It was then argued that it is natural to impose the simplifying requirements $z_{1}=0=z_{2}$, so that $Q$ is proportional to the fourth power of the (effective) radial momentum $p_{r}$. With these conditions, the solution is unique at the $3 \mathrm{PN}$ level, and is still remarkably simple, namely

$$
\widetilde{a}_{4}=a_{4} \nu, \widetilde{d}_{3}=2(3 \nu-26) \nu, \alpha_{3}=0, z_{3}=2(4-3 \nu) \nu .
$$

Here, $a_{4}$ denotes the number

$$
a_{4}=\frac{94}{3}-\frac{41}{32} \pi^{2} \simeq 18.6879027,
$$

while $\widetilde{d}_{3}$ denotes the coefficient of $\left(G M / c^{2} R\right)^{3}$ in the PN expansion of the combined metric coefficient

$$
D(R) \equiv A(R) B(R) .
$$

Replacing $B(R)$ by $D(R)$ is convenient because (as was mentioned above), in the test-mass limit $\nu \rightarrow 0$, the effective metric must reduce to the 
Schwarzschild metric, namely

$$
A(R ; \nu=0)=B^{-1}(R ; \nu=0)=1-2\left(\frac{G M}{c^{2} R}\right),
$$

so that

$$
D(R ; \nu=0)=1
$$

The final result is that the three EOB potentials $A, D, Q$ describing the $3 \mathrm{PN}$ two-body dynamics are given by the following very simple results. In terms of the EOB "gravitational potential"

$$
\begin{gathered}
u \equiv \frac{G M}{c^{2} R} \\
A_{3 \mathrm{PN}}(R)=1-2 u+2 \nu u^{3}+a_{4} \nu u^{4}, \\
D_{3 \mathrm{PN}}(R) \equiv(A(R) B(R))_{3 \mathrm{PN}}=1-6 \nu u^{2}+2(3 \nu-26) \nu u^{3}, \\
Q_{3 \mathrm{PN}}(\boldsymbol{q}, \boldsymbol{p})=\frac{1}{c^{2}} 2(4-3 \nu) \nu u^{2} \frac{p_{r}^{4}}{\mu^{2}} .
\end{gathered}
$$

In addition, the map between the (real) center-of-mass energy of the binary system $E_{\text {real }}^{\text {relative }}=H^{\text {relative }}=\mathcal{E}_{\text {relative }}^{\text {tot }}-M c^{2}$ and the effective one $\mathcal{E}_{\text {eff }}$ is found to have the very simple (but non trivial) form

$$
\frac{\mathcal{E}_{\text {eff }}}{\mu c^{2}}=1+\frac{E_{\text {real }}^{\text {relative }}}{\mu c^{2}}\left(1+\frac{\nu}{2} \frac{E_{\text {real }}^{\text {relative }}}{\mu c^{2}}\right)=\frac{s-m_{1}^{2} c^{4}-m_{2}^{2} c^{4}}{2 m_{1} m_{2} c^{4}}
$$

where $s=\left(\mathcal{E}_{\text {real }}^{\text {tot }}\right)^{2} \equiv\left(M c^{2}+E_{\text {real }}^{\text {relative }}\right)^{2}$ is Mandelstam's invariant $s=$ $-\left(p_{1}+p_{2}\right)^{2}$.

It is truly remarkable that the EOB formalism succeeds in condensing the complicated, original 3PN Hamiltonian, Eqs. (47)-(7), into the very simple potentials $A, D$ and $Q$ displayed above, together with the simple energy map Eq. (21). For instance, at the 1PN level, the already somewhat involved Lorentz-Droste-Einstein-Infeld-Hoffmann 1PN dynamics (Eqs. (4) and (5)) is simply described, within the EOB formalism, as a test particle of mass $\mu$ moving in an external Schwarzschild background of mass $M=m_{1}+m_{2}$, together with the (crucial but quite simple) energy transformation (21). [Indeed, the $\nu$-dependent corrections to $A$ and $D$ start only at the $2 \mathrm{PN}$ level.] At the $2 \mathrm{PN}$ level, the seven rather complicated $\nu$-dependent coefficients of $\widehat{H}_{2 \mathrm{PN}}(\boldsymbol{q}, \boldsymbol{p})$, Eq. (6), get condensed into the two very simple additional contributions $+2 \nu u^{3}$ in $A(u)$, and $-6 \nu u^{2}$ in $D(u)$. At the $3 \mathrm{PN}$ level, the eleven quite complicated $\nu$-dependent coefficients of $\widehat{H}_{3 \mathrm{PN}}$, Eq. (7), get condensed into only three 
simple contributions: $+a_{4} \nu u^{4}$ in $A(u),+2(3 \nu-26) \nu u^{3}$ in $D(u)$, and $Q_{3 \mathrm{PN}}$ given by Eq. (20). This simplicity of the EOB results is not only due to the reformulation of the PN-expanded Hamiltonian into an effective dynamics. Indeed, the $A$-potential happens to be much simpler that it could a priori have been: (i) as already noted it is not modified at the $1 \mathrm{PN}$ level, while one would a priori expect to have found a $1 \mathrm{PN}$ potential $A_{1 \mathrm{PN}}(u)=1-2 u+\nu a_{2} u^{2}$ with some non zero $a_{2}$; and (ii) there are striking cancellations taking place in the calculation of the $2 \mathrm{PN}$ and $3 \mathrm{PN}$ coefficients $\widetilde{a}_{2}(\nu)$ and $\widetilde{a}_{3}(\nu)$, which were a priori of the form $\widetilde{a}_{2}(\nu)=a_{2} \nu+a_{2}^{\prime} \nu^{2}$, and $\widetilde{a}_{3}(\nu)=a_{3} \nu+a_{3}^{\prime} \nu^{2}+a_{3}^{\prime \prime} \nu^{3}$, but for which the $\nu$-nonlinear contributions $a_{2}^{\prime} \nu^{2}, a_{3}^{\prime} \nu^{2}$ and $a_{3}^{\prime \prime} \nu^{3}$ precisely cancelled out.

The fact that the $3 \mathrm{PN}$ coefficient $a_{4}$ in the crucial 'effective radial potential' $A_{3 \mathrm{PN}}(R)$, Eq. (18), is rather large and positive indicates that the $\nu$-dependent nonlinear gravitational effects lead, for comparable masses $\left(\nu \sim \frac{1}{4}\right)$, to a last stable (circular) orbit (LSO) which has a higher frequency and a larger binding energy than what a naive scaling from the test-particle limit $(\nu \rightarrow 0)$ would suggest. Actually, the PN-expanded form (18) of $A_{3 \mathrm{PN}}(R)$ does not seem to be a good representation of the (unknown) exact function $A_{\mathrm{EOB}}(R)$ when the (Schwarzschild-like) relative coordinate $R$ becomes smaller than about $6 G M / c^{2}$ (which is the radius of the LSO in the test-mass limit). By continuity with the test-mass case, one a priori expects that $A_{3 \mathrm{PN}}(R)$ always exhibits a simple zero defining an EOB "effective horizon" that is smoothly connected to the Schwarzschild event horizon at $R=2 G M / c^{2}$ when $\nu \rightarrow 0$. However, the large value of the $a_{4}$ coefficient does actually prevent $A_{3 \mathrm{PN}}$ to have this property when $\nu$ is too large, and in particular when $\nu=1 / 4$. It was therefore suggested 61] to further resum6 $A_{3 \mathrm{PN}}(R)$ by replacing it by a suitable Padé $(P)$ approximant. For instance, the replacement of $A_{3 \mathrm{PN}}(R)$ by 7

$$
A_{3}^{1}(R) \equiv P_{3}^{1}\left[A_{3 \mathrm{PN}}(R)\right]=\frac{1+n_{1} u}{1+d_{1} u+d_{2} u^{2}+d_{3} u^{3}}
$$

ensures that the $\nu=\frac{1}{4}$ case is smoothly connected with the $\nu=0$ limit.

The same kind of $\nu$-continuity argument, discussed so far for the $A$ function, needs to be applied also to the $D_{3 \mathrm{PN}}(R)$ function defined in

6 The PN-expanded EOB building blocks $A_{3 \mathrm{PN}}(R), B_{3 \mathrm{PN}}(R), \ldots$ already represent a resummation of the $\mathrm{PN}$ dynamics in the sense that they have "condensed" the many terms of the original PN-expanded Hamiltonian within a very concise format. But one should not refrain to further resum the EOB building blocks themselves, if this is physically motivated.

7 We recall that the coefficients $n_{1}$ and $\left(d_{1}, d_{2}, d_{3}\right)$ of the $(1,3)$ Padé approximant $P_{3}^{1}\left[A_{3 \mathrm{PN}}(u)\right]$ are determined by the condition that the first four terms of the Taylor expansion of $A_{3}^{1}$ in powers of $u=G M /\left(c^{2} R\right)$ coincide with $A_{3 \mathrm{PN}}$. 
Eq. (19). A straightforward way to ensure that the $D$ function stays positive when $R$ decreases (since it is $D=1$ when $\nu \rightarrow 0$ ) is to replace $D_{3 \mathrm{PN}}(R)$ by $D_{3}^{0}(R) \equiv P_{3}^{0}\left[D_{3 \mathrm{PN}}(R)\right]$, where $P_{3}^{0}$ indicates the $(0,3)$ Padé approximant and explicitly reads

$$
D_{3}^{0}(R)=\frac{1}{1+6 \nu u^{2}-2(3 \nu-26) \nu u^{3}} .
$$

\section{EOB description of radiation reaction and of the emitted waveform during inspiral}

In the previous Section we have described how the EOB method encodes the conservative part of the relative orbital dynamics into the dynamics of an 'effective' particle. Let us now briefly discuss how to complete the EOB dynamics by defining some resummed expressions describing radiation reaction effects, and the corresponding waveform emitted at infinity. One is interested in circularized binaries, which have lost their initial eccentricity under the influence of radiation reaction. For such systems, it is enough (in first approximation [41; see, however, the recent results of Bini and Damour [15]) to include a radiation reaction force in the $P_{\varphi}$ equation of motion only. More precisely, we are using phase space variables $R, P_{R}, \varphi, P_{\varphi}$ associated to polar coordinates (in the equatorial plane $\theta=\frac{\pi}{2}$ ). Actually it is convenient to replace the radial momentum $P_{R}$ by the momentum conjugate to the 'tortoise' radial coordinate $R_{*}=\int d R(B / A)^{1 / 2}$, i.e. $P_{R_{*}}=(A / B)^{1 / 2} P_{R}$. The real EOB Hamiltonian is obtained by first solving Eq. (21) to get $H_{\text {real }}^{\text {total }}=\sqrt{s}$ in terms of $\mathcal{E}_{\text {eff }}$, and then by solving the effective Hamilton-Jacobi equation to get $\mathcal{E}_{\text {eff }}$ in terms of the effective phase space coordinates $\boldsymbol{q}_{\text {eff }}$ and $\boldsymbol{p}_{\text {eff }}$. The result is given by two nested square roots (we henceforth set $c=1$ ):

$$
\hat{H}_{\mathrm{EOB}}\left(r, p_{r_{*}}, \varphi\right)=\frac{H_{\mathrm{EOB}}^{\mathrm{real}}}{\mu}=\frac{1}{\nu} \sqrt{1+2 \nu\left(\hat{H}_{\mathrm{eff}}-1\right)},
$$

where

$$
\hat{H}_{\mathrm{eff}}=\sqrt{p_{r_{*}}^{2}+A(r)\left(1+\frac{p_{\varphi}^{2}}{r^{2}}+z_{3} \frac{p_{r_{*}}^{4}}{r^{2}}\right)},
$$

with $z_{3}=2 \nu(4-3 \nu)$. Here, we are using suitably rescaled dimensionless (effective) variables: $r=R / G M, p_{r_{*}}=P_{R_{*}} / \mu, p_{\varphi}=P_{\varphi} / \mu G M$, as well as a rescaled time $t=T / G M$. This leads to equations of motion for $\left(r, \varphi, p_{r_{*}}, p_{\varphi}\right)$ of the form

$$
\frac{d \varphi}{d t}=\frac{\partial \hat{H}_{\mathrm{EOB}}}{\partial p_{\varphi}} \equiv \Omega
$$




$$
\begin{aligned}
\frac{d r}{d t} & =\left(\frac{A}{B}\right)^{1 / 2} \frac{\partial \hat{H}_{\mathrm{EOB}}}{\partial p_{r_{*}}} \\
\frac{d p_{\varphi}}{d t} & =\hat{\mathcal{F}}_{\varphi} \\
\frac{d p_{r_{*}}}{d t} & =-\left(\frac{A}{B}\right)^{1 / 2} \frac{\partial \hat{H}_{\mathrm{EOB}}}{\partial r}
\end{aligned}
$$

which explicitly read

$$
\begin{aligned}
\frac{d \varphi}{d t}= & \frac{A p_{\varphi}}{\nu r^{2} \hat{H} \hat{H}_{\mathrm{eff}}} \equiv \Omega, \\
\frac{d r}{d t}= & \left(\frac{A}{B}\right)^{1 / 2} \frac{1}{\nu \hat{H} \hat{H}_{\mathrm{eff}}}\left(p_{r_{*}}+z_{3} \frac{2 A}{r^{2}} p_{r_{*}}^{3}\right), \\
\frac{d p_{\varphi}}{d t}= & \hat{\mathcal{F}}_{\varphi}, \\
\frac{d p_{r_{*}}}{d t}= & -\left(\frac{A}{B}\right)^{1 / 2} \frac{1}{2 \nu \hat{H} \hat{H}_{\mathrm{eff}}} \\
& \left\{A^{\prime}+\frac{p_{\varphi}^{2}}{r^{2}}\left(A^{\prime}-\frac{2 A}{r}\right)+z_{3}\left(\frac{A^{\prime}}{r^{2}}-\frac{2 A}{r^{3}}\right) p_{r_{*}}^{4}\right\},
\end{aligned}
$$

where $A^{\prime}=d A / d r$. As explained above the EOB metric function $A(r)$ is defined by Padé resumming the Taylor-expanded result (12) obtained from the matching between the real and effective energy levels (as we were mentioning, one uses a similar Padé resumming for $D(r) \equiv A(r) B(r)$ ). One similarly needs to resum $\hat{\mathcal{F}}_{\varphi}$, i.e., the $\varphi$ component of the radiation reaction which has been introduced on the r.h.s. of Eq. (28).

Several methods have been tried during the development of the EOB formalism to resum the radiation reaction $\widehat{\mathcal{F}}_{\varphi}$ (starting from the highorder PN-expanded results that have been obtained in the literature; see references in the Introduction above). Here, we shall briefly explain the new, parameter-free resummation technique for the multipolar waveform (and thus for the energy flux) introduced in Ref. 64, 66] and perfected in [57. To be precise, the new results discussed in Ref. [57 are twofold: on the one hand, that work generalized the $\ell=m=2$ resummed factorized waveform of 64, 66 to higher multipoles by using the most accurate currently known PN-expanded results [92, 14, 31] as well as the higher PN terms which are known in the test-mass limit [113, 114]; on the other hand, it introduced a further resummation procedure which consists in considering a new theoretical quantity, denoted as $\rho_{\ell m}(x)$, which enters the $(\ell, m)$ waveform (together with other building blocks, see below) only through its $\ell$-th power: $h_{\ell m} \propto\left(\rho_{\ell m}(x)\right)^{\ell}$. Here, and below, 
$x$ denotes the invariant $\mathrm{PN}$-ordering parameter given during inspiral by $x \equiv\left(G M \Omega / c^{3}\right)^{2 / 3}$.

The main novelty introduced by Refs. 64, 66, 57, is to write the $(\ell, m)$ multipolar waveform emitted by a circular nonspinning compact binary as the product of several factors, namely

$$
h_{\ell m}^{(\epsilon)}=\frac{G M \nu}{c^{2} R} n_{\ell m}^{(\epsilon)} c_{l+\epsilon}(\nu) x^{(\ell+\epsilon) / 2} Y^{\ell-\epsilon,-m}\left(\frac{\pi}{2}, \Phi\right) \hat{S}_{\mathrm{eff}}^{(\epsilon)} T_{\ell m} e^{\mathrm{i} \delta_{\ell m}} \rho_{\ell m}^{\ell} .
$$

Here $\epsilon$ denotes the parity of $\ell+m(\epsilon=\pi(\ell+m))$, i.e. $\epsilon=0$ for "even-parity" (mass-generated) multipoles $(\ell+m$ even $)$, and $\epsilon=1$ for "odd-parity" (current-generated) ones $(\ell+m$ odd $) ; n_{\ell m}^{(\epsilon)}$ and $c_{l+\epsilon}(\nu)$ are numerical coefficients; $\hat{S}_{\text {eff }}^{(\epsilon)}$ is a $\mu$-normalized effective source (whose definition comes from the EOB formalism); $T_{\ell m}$ is a resummed version 64,66 of an infinite number of "leading logarithms" entering the tail effects [23, 18, $\delta_{\ell m}$ is a supplementary phase (which corrects the phase effects not included in the complex tail factor $\left.T_{\ell m}\right)$, and, finally, $\left(\rho_{\ell m}\right)^{\ell}$ denotes the $\ell$-th power of the quantity $\rho_{\ell m}$ which is the new building block introduced in [57]. Note that in previous papers [64, 66] the quantity $\left(\rho_{\ell m}\right)^{\ell}$ was denoted as $f_{\ell m}$ and we will often use this notation below. Before introducing explicitly the various elements entering the waveform (34) it is convenient to decompose $h_{\ell m}$ as

$$
h_{\ell m}^{(\epsilon)}=h_{\ell m}^{(N, \epsilon)} \hat{h}_{\ell m}^{(\epsilon)}
$$

where $h_{\ell m}^{(N, \epsilon)}$ is the Newtonian contribution (i.e. the product of the first five factors in Eq. (34)) and

$$
\hat{h}_{\ell m}^{(\epsilon)} \equiv \hat{S}_{\text {eff }}^{(\epsilon)} T_{\ell m} e^{\mathrm{i} \delta_{\ell \mathrm{m}}} f_{\ell m}
$$

represents a resummed version of all the PN corrections. The PN correcting factor $\hat{h}_{\ell m}^{(\epsilon)}$, as well as all its building blocks, has the structure $\hat{h}_{\ell m}^{(\epsilon)}=1+\mathcal{O}(x)$.

The reader will find in Ref. [57] the definitions of the quantities entering the "Newtonian" waveform $h_{\ell m}^{(N, \epsilon)}$, as well as the precise definition of the effective source factor $\widehat{S}_{\text {eff }}^{(\epsilon)}$, which constitutes the first factor in the PNcorrecting factor $\widehat{h}_{\ell m}^{(\epsilon)}$. Let us only note here that the definition of $\widehat{S}_{\text {eff }}^{(\epsilon)}$ makes use of EOB-defined quantities. For instance, for even-parity waves $(\epsilon=0) \widehat{S}_{\text {eff }}^{(0)}$ is defined as the $\mu$-scaled effective energy $\mathcal{E}_{\text {eff }} / \mu c^{2}$. [We use the "J-factorization" definition of $\widehat{S}_{\text {eff }}^{(\epsilon)}$ when $\epsilon=1$, i.e. for odd parity waves.]

The second building block in the factorized decomposition is the "tail factor" $T_{\ell m}$ (introduced in Refs. [64, 66]). As mentioned above, $T_{\ell m}$ is a 
resummed version of an infinite number of "leading logarithms" entering the transfer function between the near-zone multipolar wave and the farzone one, due to tail effects linked to its propagation in a Schwarzschild background of mass $M_{\mathrm{ADM}}=H_{\mathrm{EOB}}^{\mathrm{real}}$. Its explicit expression reads

$$
T_{\ell m}=\frac{\Gamma(\ell+1-2 \mathrm{i} \hat{\hat{k}})}{\Gamma(\ell+1)} e^{\pi \hat{\hat{k}}} e^{2 \mathrm{i} \hat{\hat{k}} \log \left(2 k r_{0}\right)},
$$

where $r_{0}=2 G M / \sqrt{e}$ and $\hat{\hat{k}} \equiv G H_{\mathrm{EOB}}^{\mathrm{real}} m \Omega$ and $k \equiv m \Omega$. Note that $\hat{\hat{k}}$ differs from $k$ by a rescaling involving the real (rather than the effective) EOB Hamiltonian, computed at this stage along the sequence of circular orbits.

The tail factor $T_{\ell m}$ is a complex number which already takes into account some of the dephasing of the partial waves as they propagate out from the near zone to infinity. However, as the tail factor only takes into account the leading logarithms, one needs to correct it by a complementary dephasing term, $e^{\mathrm{i} \delta_{\ell m}}$, linked to subleading logarithms and other effects. This subleading phase correction can be computed as being the phase $\delta_{\ell m}$ of the complex ratio between the PN-expanded $\hat{h}_{\ell m}^{(\epsilon)}$ and the above defined source and tail factors. In the comparable-mass case $(\nu \neq 0)$, the $3 \mathrm{PN} \delta_{22}$ phase correction to the leading quadrupolar wave was originally computed in Ref. 66] (see also Ref. 64 for the $\nu=0$ limit). Full results for the subleading partial waves to the highest possible PN-accuracy by starting from the currently known $3 \mathrm{PN}$-accurate $\nu$-dependent waveform [31] have been obtained in [57]. For higher-order test-mass $(\nu \rightarrow 0)$ contributions, see 83,82 . For extensions of the (non spinning) factorized waveform of [57] see [102, 100, 115].

The last factor in the multiplicative decomposition of the multipolar waveform can be computed as being the modulus $f_{\ell m}$ of the complex ratio between the PN-expanded $\hat{h}_{\ell m}^{(\epsilon)}$ and the above defined source and tail factors. In the comparable mass case $(\nu \neq 0)$, the $f_{22}$ modulus correction to the leading quadrupolar wave was computed in Ref. 66. (see also Ref. 64 for the $\nu=0$ limit). For the subleading partial waves, Ref. 57] explicitly computed the other $f_{\ell m}$ 's to the highest possible PNaccuracy by starting from the currently known $3 \mathrm{PN}$-accurate $\nu$-dependent waveform [31. In addition, as originally proposed in Ref. 66], to reach greater accuracy the $f_{\ell m}(x ; \nu)$ 's extracted from the $3 \mathrm{PN}$-accurate $\nu \neq 0$ results are completed by adding higher order contributions coming from the $\nu=0$ results $\left[113,114\right.$. In the particular $f_{22}$ case discussed in 66, this amounted to adding $4 \mathrm{PN}$ and $5 \mathrm{PN} \nu=0$ terms. This "hybridization" procedure was then systematically pursued for all the other multipoles, 
using the 5.5PN accurate calculation of the multipolar decomposition of the gravitational wave energy flux of Refs. [113, 114.

The decomposition of the total PN-correction factor $\hat{h}_{\ell m}^{(\epsilon)}$ into several factors is in itself a resummation procedure which already improves the convergence of the PN series one has to deal with: indeed, one can see that the coefficients entering increasing powers of $x$ in the PN expansion of the $f_{\ell m}$ 's tend to be systematically smaller than the coefficients appearing in the usual PN expansion of $\hat{h}_{\ell m}^{(\epsilon)}$. The reason for this is essentially twofold: (i) the factorization of $T_{\ell m}$ has absorbed powers of $m \pi$ which contributed to make large coefficients in $\hat{h}_{\ell m}^{(\epsilon)}$, and (ii) the factorization of either $\hat{H}_{\text {eff }}$ or $\hat{j}$ has (in the $\nu=0$ case) removed the presence of an inverse square-root singularity located at $x=1 / 3$ which caused the coefficient of $x^{n}$ in any PN-expanded quantity to grow as $3^{n}$ as $n \rightarrow \infty$.

To further improve the convergence of the waveform several resummations of the factor $f_{\ell m}(x)=1+c_{1}^{\ell m} x+c_{2}^{\ell m} x^{2}+\ldots$ have been suggested. First, Refs. 64, 66] proposed to further resum the $f_{22}(x)$ function via a Padé $(3,2)$ approximant, $P_{2}^{3}\left\{f_{22}(x ; \nu)\right\}$, so as to improve its behavior in the strong-field-fast-motion regime. Such a resummation gave an excellent agreement with numerically computed waveforms, near the end of the inspiral and during the beginning of the plunge, for different mass ratios 64, 72, 71. As we were mentioning above, a new route for resumming $f_{\ell m}$ was explored in Ref. [57. It is based on replacing $f_{\ell m}$ by its $\ell$-th root, say

$$
\rho_{\ell m}(x ; \nu)=\left[f_{\ell m}(x ; \nu)\right]^{1 / \ell} .
$$

The basic motivation for replacing $f_{\ell m}$ by $\rho_{\ell m}$ is the following: the leading "Newtonian-level" contribution to the waveform $h_{\ell m}^{(\epsilon)}$ contains a factor $\omega^{\ell} r_{\text {harm }}^{\ell} v^{\epsilon}$ where $r_{\text {harm }}$ is the harmonic radial coordinate used in the MPM formalism [22, 56]. When computing the PN expansion of this factor one has to insert the PN expansion of the (dimensionless) harmonic radial coordinate $r_{\text {harm }}, r_{\text {harm }}=x^{-1}\left(1+c_{1} x+\mathcal{O}\left(x^{2}\right)\right)$, as a function of the gauge-independent frequency parameter $x$. The PN re-expansion of $\left[r_{\text {harm }}(x)\right]^{\ell}$ then generates terms of the type $x^{-\ell}\left(1+\ell c_{1} x+\ldots.\right)$. This is one (though not the only one) of the origins of $1 \mathrm{PN}$ corrections in $h_{\ell m}$ and $f_{\ell m}$ whose coefficients grow linearly with $\ell$. The study of [57] has pointed out that these $\ell$-growing terms are problematic for the accuracy of the $\mathrm{PN}$-expansions. The replacement of $f_{\ell m}$ by $\rho_{\ell m}$ is a cure for this problem.

Several studies, both in the test-mass limit, $\nu \rightarrow 0$ (see Fig. 1 in [57]) and in the comparable-mass case (see notably Fig. 4 in [67]), have shown that the resummed factorized (inspiral) EOB waveforms defined above provided remarkably accurate analytical approximations to the "exact" inspiral waveforms computed by numerical simulations. These resummed 
multipolar EOB waveforms are much closer (especially during late inspiral) to the exact ones than the standard $\mathrm{PN}$-expanded waveforms given by Eq. (35) with a PN-correction factor of the usual "Taylor-expanded" form

$$
\widehat{h}_{\ell m}^{(\epsilon) \mathrm{PN}}=1+c_{1}^{\ell m} x+c_{3 / 2}^{\ell m} x^{3 / 2}+c_{2}^{\ell m} x^{2}+\ldots
$$

See Fig. 1 in [57, and slide 29 in my (June 2012) Prague presentation.

Finally, one uses the newly resummed multipolar waveforms (34) to define a resummation of the radiation reaction force $\mathcal{F}_{\varphi}$ as

$$
\mathcal{F}_{\varphi} \equiv-\frac{1}{\Omega} F^{\left(\ell_{\max }\right)},
$$

where the (instantaneous, circular) GW flux $F^{\left(\ell_{\max }\right)}$ is defined as

$$
F^{\left(\ell_{\max }\right)} \equiv \frac{2}{16 \pi G} \sum_{\ell=2}^{\ell_{\max }} \sum_{m=1}^{\ell}(m \Omega)^{2}\left|R h_{\ell m}\right|^{2} .
$$

Summarizing: Eqs. (34) and (39), (40) define resummed EOB versions of the waveform $h_{\ell m}$, and of the radiation reaction $\widehat{\mathcal{F}}_{\varphi}$, during inspiral. A crucial point is that these resummed expressions are parameter-free. Given some current approximation to the conservative EOB dynamics (i.e. some expressions for the $A, D, Q$ potentials) they complete the EOB formalism by giving explicit predictions for the radiation reaction (thereby completing the dynamics, see Eqs. (26)-(29)), and for the emitted inspiral waveform.

\section{EOB description of the merger of binary black holes and of the ringdown of the final black hole}

Up to now we have reviewed how the EOB formalism, starting only from analytical information obtained from PN theory, and adding extra resummation requirements (both for the EOB conservative potentials $A$, Eq. (22), and D, Eq. (23), and for the waveform, Eq. (34), and its associated radiation reaction force, Eqs. (39), (40)) makes specific predictions, both for the motion and the radiation of binary black holes. The analytical calculations underlying such an EOB description are essentially based on skeletonizing the two black holes as two, sufficiently separated point masses, and therefore seem unable to describe the merger of the two black holes, and the subsequent ringdown of the final, single black hole formed during the merger. However, as early as 2000 [41, the EOB formalism went one step further and proposed a specific strategy for describing the complete waveform emitted during the entire coalescence 
process, covering inspiral, merger and ringdown. This EOB proposal is somewhat crude. However, the predictions it has made (years before NR simulations could accurately describe the late inspiral and merger of binary black holes) have been broadly confirmed by subsequent NR simulations. [See the Introduction for a list of EOB predictions.] The original EOB proposal (which was motivated partly by the closeness between the $2 \mathrm{PN}$ accurate effective metric $g_{\mu \nu}^{\text {eff }}[40$ and the Schwarzschild metric, and by the results of Refs. [77] and [109]) consists of:

(i) defining, within EOB theory, the instant of (effective) "merger" of the two black holes as the (dynamical) EOB time $t_{m}$ where the orbital frequency $\Omega(t)$ reaches its maximum;

(ii) describing (for $t \leq t_{m}$ ) the inspiral-plus-plunge (or simply insplunge) waveform, $h^{\text {insplunge }}(t)$, by using the inspiral EOB dynamics and waveform reviewed in the previous Section; and

(iii) describing (for $t \geq t_{m}$ ) the merger-plus-ringdown waveform as a superposition of several quasi-normal-mode (QNM) complex frequencies of a final Kerr black hole (of mass $M_{f}$ and spin parameter $a_{f}$, self-consistency estimated within the EOB formalism), say

$$
\left(\frac{R c^{2}}{G M}\right) h_{\ell m}^{\text {ringdown }}(t)=\sum_{N} C_{N}^{+} e^{-\sigma_{N}^{+}\left(t-t_{m}\right)},
$$

with $\sigma_{N}^{+}=\alpha_{N}+i \omega_{N}$, and where the label $N$ refers to indices $\left(\ell, \ell^{\prime}, m, n\right)$, with $(\ell, m)$ being the Schwarzschild-background multipolarity of the considered (metric) waveform $h_{\ell m}$, with $n=0,1,2 \ldots$ being the 'overtone number' of the considered Kerr-background Quasi-Normal-Mode, and $\ell^{\prime}$ the degree of its associated spheroidal harmonics $S_{\ell^{\prime} m}(a \sigma, \theta)$;

(iv) determining the excitation coefficients $C_{N}^{+}$of the QNM's in Eq. (41) by using a simplified representation of the transition between plunge and ring-down obtained by smoothly matching (following Ref. 64]), on a $(2 p+1)$-toothed "comb" $\left(t_{m}-p \delta, \ldots, t_{m}-\delta, t_{m}, t_{m}+\delta, \ldots, t_{m}+p \delta\right)$ centered around the merger (and matching) time $t_{m}$, the inspiral-plusplunge waveform to the above ring-down waveform.

Finally, one defines a complete, quasi-analytical EOB waveform (covering the full process from inspiral to ring-down) as:

$$
h_{\ell m}^{\mathrm{EOB}}(t)=\theta\left(t_{m}-t\right) h_{\ell m}^{\text {insplunge }}(t)+\theta\left(t-t_{m}\right) h_{\ell m}^{\text {ringdown }}(t),
$$

where $\theta(t)$ denotes Heaviside's step function. The final result is a waveform that essentially depends only on the choice of a resummed EOB $A(u)$ potential, and, less importantly, on the choice of resummation of the main waveform amplitude factor $f_{22}=\left(\rho_{22}\right)^{2}$. 
We have emphasized here that the EOB formalism is able, in principle, starting only from the best currently known analytical information, to predict the full waveform emitted by coalescing binary black holes. The early comparisons between $3 \mathrm{PN}$-accurate EOB predicted waveform 8 and NR-computed waveforms showed a satisfactory agreement between the two, within the (then relatively large) NR uncertainties [39, 103. Moreover, as we shall discuss below, it has been recently shown that the currently known Padé-resummed $3 \mathrm{PN}$-accurate $A(u)$ potential is able, as is, to describe with remarkable accuracy several aspects of the dynamics of coalescing binary black holes, 96, 73.

On the other hand, when NR started delivering high-accuracy waveforms, it became clear that the $3 \mathrm{PN}$-level analytical knowledge incorporated in EOB theory was not accurate enough for providing waveforms agreeing with NR ones within the high-accuracy needed for detection, and data analysis of upcoming GW signals. [See, e.g., the discussion in Section II of Ref. [100.] At that point, one made use of the natural flexibility of the EOB formalism. Indeed, as already emphasized in early EOB work [47, 54, we know from the analytical point of view that there are (yet uncalculated) further terms in the $u$-expansions of the EOB potentials $A(u), D(u), \ldots$ (and in the $x$-expansion of the waveform), so that these terms can be introduced either as "free parameter(s) in constructing a bank of templates, and [one should] wait until" GW observations determine their value(s) [47, or as "fitting parameters and adjusted so as to reproduce other information one has about the exact results" (to quote Ref. [54]). For instance, modulo logarithmic corrections that will be further discussed below, the Taylor expansion in powers of $u$ of the main EOB potential $A(u)$ reads

$$
A^{\text {Taylor }}(u ; \nu)=1-2 u+\widetilde{a}_{3}(\nu) u^{3}+\widetilde{a}_{4}(\nu) u^{4}+\widetilde{a}_{5}(\nu) u^{5}+\widetilde{a}_{6}(\nu) u^{6}+\ldots
$$

where the $2 \mathrm{PN}$ and $3 \mathrm{PN}$ coefficients $\widetilde{a}_{3}(\nu)=2 \nu$ and $\widetilde{a}_{4}(\nu)=a_{4} \nu$ are known, but where the $4 \mathrm{PN}, 5 \mathrm{PN}, \ldots$ coefficients, $\widetilde{a}_{5}(\nu), \widetilde{a}_{6}(\nu), \ldots$ have not yet been calculated (see, however, below). A first attempt was made in 54] to use numerical data (on circular orbits of corotating black holes) to fit for the value of a (single, effective) $4 \mathrm{PN}$ parameter of the simple form $\widetilde{a}_{5}(\nu)=a_{5} \nu$ entering a Padé-resummed $4 \mathrm{PN}$-level $A$ potential, i.e.

$$
A_{4}^{1}\left(u ; a_{5}, \nu\right)=P_{4}^{1}\left[A_{3 \mathrm{PN}}(u)+\nu a_{5} u^{5}\right] .
$$

This strategy was pursued in Ref. [42, 66 and many subsequent works. It was pointed out in Ref. 67. that the introduction of a further 5PN

8 The new, resummed EOB waveform discussed above was not available at the time, so that these comparisons employed the coarser "Newtonian-level" EOB waveform $h_{22}^{(N, \epsilon)}(x)$. 
coefficient $\widetilde{a}_{6}(\nu)=a_{6} \nu$, entering a Padé-resummed 5 PN-level $A$ potential, i.e.

$$
A_{5}^{1}\left(u ; a_{5}, a_{6}, \nu\right)=P_{5}^{1}\left[A_{3 \mathrm{PN}}(u)+\nu a_{5} u^{5}+\nu a_{6} u^{6}\right],
$$

helped in having a closer agreement with accurate NR waveforms.

In addition, Refs. [64, 66] introduced another type of flexibility parameters of the EOB formalism: the non quasi-circular (NQC) parameters accounting for uncalculated modifications of the quasi-circular inspiral waveform presented above, linked to deviations from an adiabatic quasi-circular motion. These NQC parameters are of various types, and subsequent works [72, 71, 67, 43, 12, 9, 100] have explored several ways of introducing them. They enter the EOB waveform in two separate ways. First, through an explicit, additional complex factor multiplying $h_{\ell m}, e . g$.

$$
f_{\ell m}^{\mathrm{NQC}}=\left(1+a_{1}^{\ell m} n_{1}+a_{2}^{\ell m} n_{2}\right) \exp \left[i\left(a_{3}^{\ell m} n_{3}+a_{4}^{\ell m} n_{4}\right)\right]
$$

where the $n_{i}$ 's are dynamical functions that vanish in the quasi-circular limit (with $n_{1}, n_{2}$ being time-even, and $n_{3}, n_{4}$ time-odd). For instance, one usually takes $n_{1}=\left(p_{r_{*}} / r \Omega\right)^{2}$. Second, through the (discrete) choice of the argument used during the plunge to replace the variable $x$ of the quasicircular inspiral argument: e.g. either $x_{\Omega} \equiv(G M \Omega)^{2 / 3}$, or (following [53]) $x_{\varphi} \equiv v_{\varphi}^{2}=\left(r_{\omega} \Omega\right)^{2}$ where $v_{\varphi} \equiv \Omega r_{\omega}$, and $r_{\omega} \equiv r\left[\psi\left(r, p_{\varphi}\right)\right]^{1 / 3}$ is a modified EOB radius, with $\psi$ being defined as

$$
\psi\left(r, p_{\varphi}\right)=\frac{2}{r^{2}}\left(\frac{d A(r)}{d r}\right)^{-1}\left[1+2 \nu\left(\sqrt{A(r)\left(1+\frac{p_{\varphi}^{2}}{r^{2}}\right)}-1\right)\right] .
$$

For a given value of the symmetric mass ratio, and given values of the $A$-flexibility parameters $\widetilde{a}_{5}(\nu), \widetilde{a}_{6}(\nu)$ one can determine the values of the NQC parameters $a_{i}^{\ell m}$ 's from accurate NR simulations of binary black hole coalescence (with mass ratio $\nu$ ) by imposing, say, that the complex EOB waveform $h_{\ell m}^{\mathrm{EOB}}\left(t^{\mathrm{EOB}} ; \widetilde{a}_{5}, \widetilde{a}_{6} ; a_{i}^{\ell m}\right)$ osculates the corresponding $\mathrm{NR}$ one $h_{\ell m}^{\mathrm{NR}}\left(t^{\mathrm{NR}}\right)$ at their respective instants of "merger", where $t_{\text {merger }}^{\mathrm{EOB}} \equiv t_{m}^{\mathrm{EOB}}$ was defined above (maximum of $\Omega^{\mathrm{EOB}}(t)$ ), while $t_{\text {merger }}^{\mathrm{NR}}$ is defined, say, as the (retarded) NR time where the modulus $\left|h_{22}^{\mathrm{NR}}(t)\right|$ of the quadrupolar waveform reaches its maximum. The order of osculation that one requires between $h_{\ell m}^{\mathrm{EOB}}(t)$ and $h_{\ell m}^{\mathrm{NR}}(t)$ (or, separately, between their moduli and their phases or frequencies) depends on the number of NQC parameters $a_{i}^{\ell m}$. For instance, $a_{1}^{\ell m}$ and $a_{2}^{\ell m}$ affect only the modulus of $h_{\ell m}^{\mathrm{EOB}}$ and allow one to match both $\left|h_{\ell m}^{\mathrm{EOB}}\right|$ and its first time derivative, at merger, to their NR counterparts, while $a_{3}^{\ell m}, a_{4}^{\ell m}$ affect only the phase of the EOB waveform, and allow one to match the GW frequency $\omega_{\ell m}^{\mathrm{EOB}}(t)$ and its first time derivative, at merger, to their NR counterparts. The above 
EOB/NR matching scheme has been developed and declined in various versions in Refs. [72, 71, 67, 43, 12, 13, 9, 100, 70. One has also extracted the needed matching data from accurate NR simulations, and provided explicit, analytical $\nu$-dependent fitting formulas for them [67, 100, 70.

Having so "calibrated" the values of the NQC parameters by extracting non-perturbative information from a sample of NR simulations, one can then, for any choice of the $A$-flexibility parameters, compute a full EOB waveform (from early inspiral to late ringdown). The comparison of the latter NQC-completed EOB waveform to the results of NR simulations is discussed in the next Section.

\section{EOB vs NR}

There have been several different types of comparison between EOB and NR. For instance, the early work 39 pioneered the comparison between a purely analytical EOB waveform (uncalibrated to any NR information) and a NR waveform, while the early work [65] compared the predictions for the final spin of a coalescing black hole binary made by EOB, completed by the knowledge of the energy and angular momentum lost during ringdown by an extreme mass ratio binary (computed by the test-mass NR code of [74]), to comparable-mass NR simulations [86]. Since then, many other EOB/NR comparisons have been performed, both in the comparablemass case [103, 42, 66, 72, 71, 67, 43, and in the small-mass-ratio case [64, 121, 122, 12, 9]. Note in this respect that the numerical simulations of the GW emission by extreme mass-ratio binaries have provided (and still provide) a very useful "laboratory" for learning about the motion and radiation of binary systems, and their description within the EOB formalism.

Here we shall discuss only two recent examples of EOB/NR comparisons, which illustrate different facets of this comparison.

\subsection{EOB[NR] waveforms vs NR ones}

We explained above how one could complete the EOB formalism by calibrating some of the natural EOB flexibility parameters against NR data. First, for any given mass ratio $\nu$ and any given values of the $A$-flexibility parameters $\widetilde{a}_{5}(\nu), \widetilde{a}_{6}(\nu)$, one can use NR data to uniquely determine the NQC flexibility parameters $a_{i}$ 's. In other words, we have (for a given $\nu$ )

$$
a_{i}=a_{i}\left[\mathrm{NR} \text { data } ; a_{5}, a_{6}\right],
$$

where we defined $a_{5}$ and $a_{6}$ so that $\widetilde{a}_{5}(\nu)=a_{5} \nu, \widetilde{a}_{6}(\nu)=a_{6} \nu$. [We allow for some residual $\nu$-dependence in $a_{5}$ and $a_{6}$.] Inserting these values in the 
(analytical) EOB waveform then defines an NR-completed EOB waveform which still depends on the two unknown flexibility parameters $a_{5}$ and $a_{6}$.

In Ref. 67 the $\left(a_{5}, a_{6}\right)$-dependent predictions made by such a NRcompleted EOB formalism were compared to the high-accuracy waveform from an equal-mass binary black hole $(\nu=1 / 4)$ computed by the CaltechCornell-CITA group [112], (and then made available on the web). It was found that there is a strong degeneracy between $a_{5}$ and $a_{6}$ in the sense that there is an excellent EOB-NR agreement for an extended region in the $\left(a_{5}, a_{6}\right)$-plane. More precisely, the phase difference between the EOB (metric) waveform and the Caltech-Cornell-CITA one, considered between $\mathrm{GW}$ frequencies $M \omega_{\mathrm{L}}=0.047$ and $M \omega_{\mathrm{R}}=0.31$ (i.e., the last $16 \mathrm{GW}$ cycles before merger), stays smaller than 0.02 radians within a long and thin banana-like region in the $\left(a_{5}, a_{6}\right)$-plane. This "good region" approximately extends between the points $\left(a_{5}, a_{6}\right)=(0,-20)$ and $\left(a_{5}, a_{6}\right)=(-36,+520)$. As an example (which actually lies on the boundary of the "good region"), we shall consider here (following Ref. 69]) the specific values $a_{5}=0, a_{6}=-20$ (to which correspond, when $\nu=1 / 4$, $a_{1}=-0.036347, a_{2}=1.2468$ ). [Ref. 67] did not make use of the NQC phase flexibility; i.e. it took $a_{3}=a_{4}=0$. In addition, it used $n_{2}=\ddot{r} / r \Omega^{2}$ and introduced a (real) modulus NQC factor $f_{\ell m}^{\mathrm{NQC}}$ only for the dominant quadrupolar wave $\ell=2=m$.] We henceforth use $M$ as time unit. This result relies on the proper comparison between NR and EOB time series, which is a delicate subject. In fact, to compare the NR and EOB phase time-series $\phi_{22}^{\mathrm{NR}}\left(t_{\mathrm{NR}}\right)$ and $\phi_{22}^{\mathrm{EOB}}\left(t_{\mathrm{EOB}}\right)$ one needs to shift, by additive constants, both one of the time variables, and one of the phases. In other words, we need to determine $\tau$ and $\alpha$ such that the "shifted" EOB quantities

$$
t_{\mathrm{EOB}}^{\prime}=t_{\mathrm{EOB}}+\tau, \quad \phi_{22}^{\prime \mathrm{EOB}}=\phi_{22}^{\mathrm{EOB}}+\alpha
$$

"best fit" the NR ones. One convenient way to do so is first to "pinch" (i.e. constrain to vanish) the EOB/NR phase difference at two different instants (corresponding to two different frequencies $\omega_{1}$ and $\omega_{2}$ ). Having so related the EOB time and phase variables to the NR ones we can straigthforwardly compare the EOB time series to its NR correspondant. In particular, we can compute the (shifted) EOB-NR phase difference

$$
\Delta^{\omega_{1}, \omega_{2}} \phi_{22}^{\mathrm{EOBNR}}\left(t_{\mathrm{NR}}\right) \equiv \phi_{22}^{\prime \mathrm{EOB}}\left(t^{\prime \mathrm{EOB}}\right)-\phi_{22}^{\mathrm{NR}}\left(t^{\mathrm{NR}}\right) .
$$

Figure 2 compares (the real part of) the analytical EOB metric quadrupolar waveform $\Psi_{22}^{\mathrm{EOB}} / \nu$ to the corresponding (Caltech-CornellCITA) NR metric waveform $\Psi_{22}^{\mathrm{NR}} / \nu$. [Here, $\Psi_{22}$ denotes the Zerilli-

9 The two "pinching" frequencies used for this comparison are $M \omega_{1}=0.047$ and $M \omega_{2}=0.31$. 


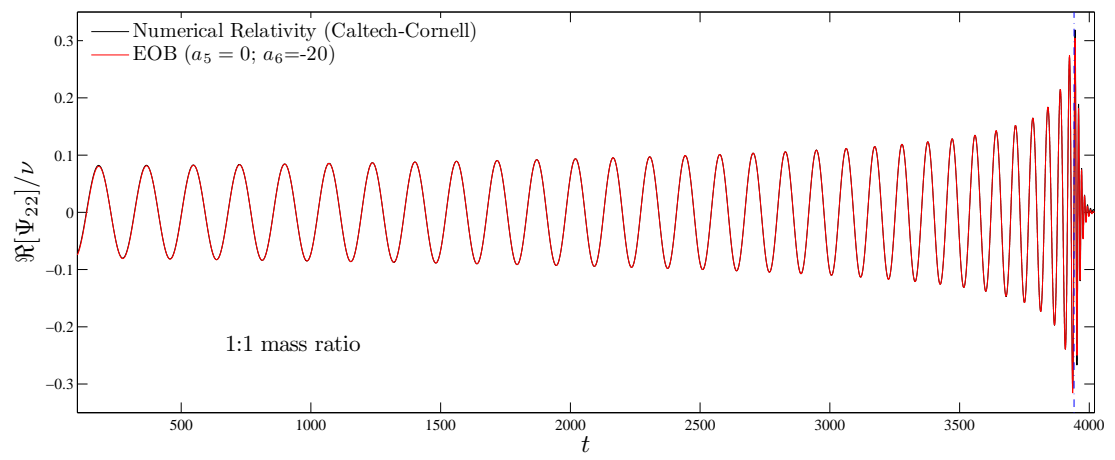

Figure 2. This figure illustrates the comparison (made in Refs. 67, 69]) between the (NR-completed) EOB waveform (Zerilli-normalized quadrupolar $(\ell=m=2)$ metric waveform (42) with parameter-free radiation reaction (39) and with $\left.a_{5}=0, a_{6}=-20\right)$ and one of the most accurate numerical relativity waveform (equal-mass case) nowadays available [112. The phase difference between the two is $\Delta \phi \leq \pm 0.01$ radians during the entire inspiral and plunge, which is at the level of the numerical error.

normalized asymptotic quadrupolar waveform, i.e. $\Psi_{22} \equiv \widehat{R} h_{22} / \sqrt{24}$ with $\widehat{R}=R c^{2} / G M$.] This NR metric waveform has been obtained by a double time-integration (following the procedure of Ref. [71) from the original, publicly available, curvature waveform $\psi_{4}^{22}$ [112. Such a curvature waveform has been extrapolated both in resolution and in extraction radius. The agreement between the analytical prediction and the NR result is striking, even around the merger. See Fig. 3 which closes up on the merger. The vertical line indicates the location of the EOBmerger time, i.e., the location of the maximum of the orbital frequency.

The phasing agreement between the waveforms is excellent over the full time span of the simulation (which covers 32 cycles of inspiral and about 6 cycles of ringdown), while the modulus agreement is excellent over the full span, apart from two cycles after merger where one can notice a difference. More precisely, the phase difference, $\Delta \phi=\phi_{\text {metric }}^{\mathrm{EOB}}-\phi_{\text {metric }}^{\mathrm{NR}}$, remains remarkably small ( $\sim \pm 0.02$ radians) during the entire inspiral and plunge $\left(\omega_{2}=0.31\right.$ being quite near the merger). By comparison, the root-sum of the various numerical errors on the phase (numerical truncation, outer boundary, extrapolation to infinity) is about 0.023 radians during the inspiral [112. At the merger, and during the ringdown, 




Figure 3. Close up around merger of the waveforms of Fig. 2] Note the excellent agreement between both modulus and phasing also during the ringdown phase.

$\Delta \phi$ takes somewhat larger values $(\sim \pm 0.1$ radians $)$, but it oscillates around zero, so that, on average, it stays very well in phase with the NR waveform whose error rises to \pm 0.05 radians during ringdown. In addition, Ref. 67] compared the EOB waveform to accurate numerical relativity data (obtained by the Jena group [71) on the coalescence of unequal mass-ratio black-hole binaries. Again, the agreement was good, and within the numerical error bars.

This type of high-accuracy comparison between NR waveforms and $\mathrm{EOB}[\mathrm{NR}]$ ones (where $\mathrm{EOB}[\mathrm{NR}]$ denotes a $\mathrm{EOB}$ formalism which has been completed by fitting some EOB-flexibility parameters to NR data) has been pursued and extended in Ref. [100. The latter reference used the "improved" EOB formalism of Ref. 67] with some variations (e.g. a third modulus NQC coefficient $a_{i}$, two phase NQC coefficients, the argument $x_{\Omega}=(M \Omega)^{2 / 3}$ in $\left(\rho_{\ell m}^{\text {Taylor }}(x)\right)^{\ell}$, eight QNM modes $)$ and calibrated it to NR simulations of mass ratios $q=m_{2} / m_{1}=1,2,3,4$ and 6 performed by the Caltech-Cornell-CITA group [37, 97. They considered not only the leading $(\ell, m)=(2,2) \mathrm{GW}$ mode, but the subleading ones $(2,1),(3,3),(4,4)$ and $(5,5)$. They found that, for this large range of mass ratios, EOB[NR] (with suitably fitted, $\nu$-dependent values of $a_{5}$ and $a_{6}$ ) was able to describe 
the NR waveforms essentially within the NR errors. This confirms the usefulness of the EOB formalism in helping the detection and analysis of upcoming GW signals.

Here, having in view GW observations from ground-based interferometric detectors we focussed on comparable-mass systems. The EOB formalism has also been compared to NR results in the extreme mass-ratio limit $\nu \ll 1$. In particular, Ref. [12] found an excellent agreement between the analytical and numerical results.

\section{2. $E O B[3 P N]$ dynamics vs $N R$ one}

Let us also mention other types of EOB/NR comparisons. Recently, two examples of EOB/NR comparisons have been performed directly at the level of the dynamics of a binary black hole, rather than at the level of the waveform. Moreover, contrary to the waveform comparisons of the previous subsection which involved an NR-completed EOB formalism ("EOB[NR]"), the dynamical comparisons we are going to discuss involve the purely analytical $3 \mathrm{PN}$-accurate EOB formalism ("EOB[3PN]"), without any NR-based improvement.

First, Le Tiec et al. 96 have extracted from accurate NR simulations of slightly eccentric binary black-hole systems (for several mass ratios $q=m_{1} / m_{2}$ between $1 / 8$ and 1 ) the function relating the periastronadvance parameter

$$
K=1+\frac{\Delta \Phi}{2 \pi},
$$

(where $\Delta \Phi$ is the periastron advance per radial period) to the dimensionless averaged angular frequency $M \Omega_{\varphi}$ (with $M=m_{1}+m_{2}$ as above). Then they compared the NR-estimate of the mass-ratio dependent functional relation

$$
K=K\left(M \Omega_{\varphi} ; \nu\right),
$$

where $\nu=q /(1+q)^{2}$, to the predictions of various analytic approximation schemes: PN theory, EOB theory and two different ways of using GSF theory. Let us only mention here that the prediction from the purely analytical EOB[3PN] formalism for $K\left(M \Omega_{\varphi} ; \nu\right)$ [48 agreed remarkably well (essentially within numerical errors) with its NR estimate for all mass ratios, while, by contrast, the PN-expanded prediction for $K\left(M \Omega_{\varphi} ; \nu\right) 60$. showed a much poorer agreement, especially as $q$ moved away from 1 .

Second, Damour, Nagar, Pollney and Reisswig [73] have recently extracted from accurate NR simulations of black-hole binaries (with mass ratios $q=m_{2} / m_{1}=1,2$ and 3) the gauge-invariant relation between the (reduced) binding energy $E=\left(\mathcal{E}^{\text {tot }}-M\right) / \mu$ and the (reduced) angular 


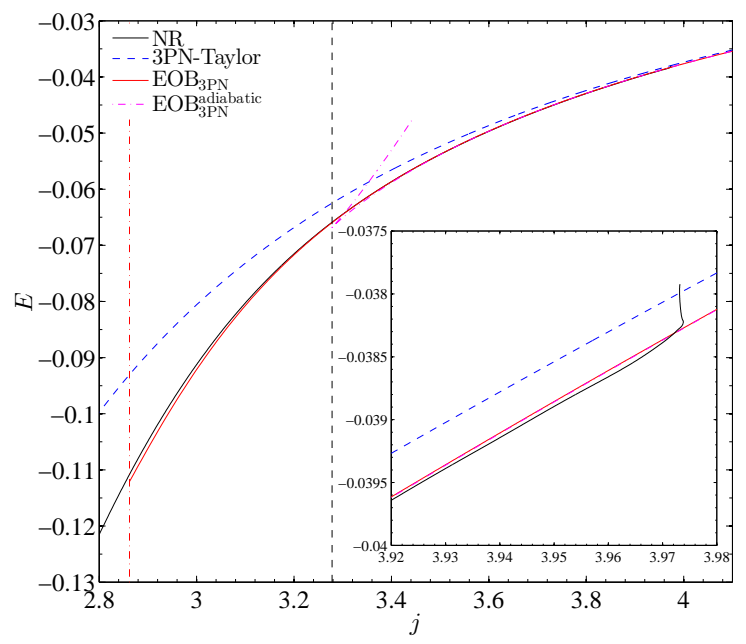

Figure 4. Comparison (made in [73]) between various analytical estimates of the energy-angular momentum functional relation and its numericalrelativity estimate (equal-mass case). The standard "Taylor-expanded" $3 \mathrm{PN} E(j)$ curve shows the largest deviation from NR results, especially at low $j$ 's, while the two (adiabatic and nonadiabatic) 3PN-accurate, non$N R$-calibrated EOB $E(j)$ curves agree remarkably well with the NR one.

momentum $j=J /(G \mu M)$ of the system. Then they compared the NRestimate of the mass-ratio dependent functional relation

$$
E=E(j ; \nu)
$$

to the predictions of various analytic approximation schemes: PN theory and various versions of EOB theory (some of these versions were NRcompleted). Let us only mention here that the prediction from the purely analytical, $3 \mathrm{PN}$-accurate $\mathrm{EOB}[3 \mathrm{PN}]$ for $E(j ; \nu)$ agreed remarkably well with its NR estimate (for all mass ratios) essentially down to the merger. This is illustrated in Fig. 4 for the $q=1$ case. By contrast, the 3PN expansion in (powers of $1 / c^{2}$ ) of the function $E(j ; \nu)$ showed a much poorer agreement (for all mass ratios). 


\section{Other developments}

\subsection{EOB with spinning bodies}

We lack space here for discussing the extension of the EOB formalism to binary systems made of spinning bodies. Let us start by mentionning that the spin-extension of the EOB formalism was initiated in Ref. 47, that the first EOB-based analytical calculation of a complete waveform from a spinning binary was performed in Ref. 38, and that the first attempt at calibrating a spinning EOB model to accurate NR simulations of spinning (non precessing) black-hole binaries was presented in 101. In addition, several formal aspects related to the inclusion of spins in the EOB formalism have been discussed in Refs. 63, 11, 7, 98, 8, (see references within these papers for $\mathrm{PN}$ works dealing with spin effects) and a generalization of the factorized multipolar waveform of Ref. [57. to spinning, non-precessing binaries has been constructed in Refs. [102 115].

\subsection{EOB with tidally deformed bodies}

In binary systems comprising neutron stars, rather than black holes, the tidal deformation of the neutron star(s) will significantly modify the phasing of the emitted gravitational waveform during the late inspiral. As GW's from binary neutron stars are expected sources for upcoming groundbased GW detectors, it is important to extend the EOB formalism by including tidal effects (see 75] and references therein). This extension has been defined in Refs. 68, 16. The comparison between this tidal-extended EOB and state-of-the-art NR simulations of neutron-star binaries has been discussed in Refs. 22, 3. It appears from these comparisons that the tidalextended EOB formalism is able to describe the motion and radiation of neutron-star binaries within NR errors. More accurate simulations will be needed to ascertain whether one needs to calibrate some higher-order flexibility parameters of the tidal-EOB formalism, or whether the currently known analytic accuracy is sufficient.

\section{3. $E O B$ and $G S F$}

We mentioned in the Introduction that GSF theory has recently opened a new source of information on the general relativistic two-body problem. Let us briefly mention here that there has been, recently, a quite useful transfer of information from GSF theory to EOB theory. The program of using GSF-theory to improve EOB-theory was first highlighted in Ref. [48. That work pointed to several concrete gauge-invariant calculations (within GSF theory) that would provide accurate information about the $O(\nu)$ contributions to several EOB potentials. More precisely, let us define the 
functions $a(u)$ and $\bar{d}(u)$ as the $\nu$-linear contributions to the EOB potentials $A(u ; \nu)$ and $\bar{D}(u ; \nu) \equiv D^{-1}(u ; \nu)$ :

$$
\begin{gathered}
A(u ; \nu)=1-2 u+\nu a(u)+O\left(\nu^{2}\right), \\
\bar{D}(u ; \nu)=(A B)^{-1}=1+\nu \bar{d}(u)+O\left(\nu^{2}\right) .
\end{gathered}
$$

Ref. 48. has shown that a computation of the GSF-induced correction to the periastron advance of slightly eccentric orbits would allow one to compute the following combination of EOB functions

$$
\bar{\rho}(u)=a(u)+u a^{\prime}(u)+\frac{1}{2} u(1-2 u) a^{\prime \prime}(u)+(1-6 u) \bar{d}(u) .
$$

The GSF-calculation of the EOB function $\bar{\rho}(u)$ was then performed in Ref. [6] (in the range $0 \leq u \leq \frac{1}{6}$ ).

More recently, a series of works by Le Tiec and collaborators 95, 94, 10 . have (through an indirect route) shown how GSF calculations could be used to compute the EOB $\nu$-linear $a(u)$ function separately from the $\bar{d}(u)$ one. Ref. 10] then gave a fitting formula for $a(u)$ over the interval $0 \leq u \leq \frac{1}{5}$ as well as accurate estimates of the coefficients of the Taylor expansion of $a(u)$ around $u=0$ (corresponding to the knowledge of the PN expansion of $a(u)$ to a very high PN order). Very recently, Ackay et al. [1] succeeded in accurately computing (through GSF theory) the EOB $a(u)$ function over the larger interval $0 \leq u \leq \frac{1}{3}$. It was (surprisingly) found that $a(u)$ diverges like $a(u) \approx 0.25(1-3 u)^{-1 / 2}$ at the light-ring limit $u \rightarrow\left(\frac{1}{3}\right)^{-}$. The meaning for EOB theory of this singular behavior of $a(u)$ at the light-ring is discussed in detail in Ref. [1.

\subsection{Toward further improvements to EOB}

Let us finally mention some avenues for further progress in EOB theory.

Logarithmic contributions to the $A(u)$ and $\bar{D}(u)$ functions have been recently computed at the $4 \mathrm{PN}$ level [48, 29] and even the $5 \mathrm{PN}$ one [49, 10. They have been incorporated in a recent, improved implementation of the EOB formalism [70.

Two groups have embarked on a calculation of the (full) conservative dynamics at the $4 \mathrm{PN}$ level $[81,91$. If they succeed, it will be important to translate their gauge-dependent results in the gauge-invariant form used in EOB theory. [Remember that EOB theory is essentially based on the gauge-invariant Delaunay Hamiltonian $H\left(I_{a}\right)$.]

More generally, let us emphasize that the EOB formalism provides a convenient, gauge-invariant way of packaging both the conservative 
dynamics and the multipolar waveform. This EOB packaging has often turned out to be very economical. We recommend that authors computing high-order $\mathrm{PN}$ corrections to either the dynamics or the waveform reexpress their results in terms of the EOB building blocks.

For instance, Jaranowski and Schaëfer 91 have recently given a partial result at $4 \mathrm{PN}$, expressed in terms of the (gauge-invariant) function $E\left(M \Omega_{\varphi} ; \nu\right)$. In terms of this function, the $4 \mathrm{PN}$ contribution is a polynomial of the fourth degree in $\nu$, namely, with $x \equiv\left(M \Omega_{\varphi}\right)^{2 / 3}$ and

$$
\begin{aligned}
E(x ; \nu)= & -\frac{1}{2} \mu c^{2} x\left(1+e_{1 P N}(\nu) x+e_{2 P N}(\nu) x^{2}+e_{3 P N}(\nu) x^{3}\right. \\
& \left.+e_{4 P N}(\nu ; \ln x) x^{4}+O\left(x^{5} \ln x\right)\right),
\end{aligned}
$$

they found

$$
e_{4 P N}(\nu ; \ln x)=-\frac{3969}{128}+c_{1} \nu+c_{2} \nu^{2}+\frac{301}{1728} \nu^{3}+\frac{77}{31104} \nu^{4}+\frac{448}{15} \nu \ln x,
$$

where they could not compute the values of the coefficients $c_{1}$ and $c_{2}$ of the terms linear and quadratic in $\nu$, but only the contributions cubic and quartic in $\nu$. We wish to point out that their result is re-expressed in a more economical (and more informative) way in terms of the basic EOB potential $A(u ; \nu)$. Indeed, in terms of the $\mathrm{PN}$ expansion, of $A(u ; \nu)$,

$A^{\text {Taylor }}(u ; \nu)=1-2 u+\widetilde{a}_{3}(\nu) u^{3}+\widetilde{a}_{4}(\nu) u^{4}+\widetilde{a}_{5}(\nu ; \ln u) u^{5}+\widetilde{a}_{6}(\nu ; \ln u) u^{6}+\ldots$

the information contained in the above result can be entirely re-expressed in terms of the $4 \mathrm{PN}$-level coefficient $\widetilde{a}_{5}(\nu ; \ln u)$. When doing this reexpression, one then finds that the information content of Eq. (48) is that the $4 \mathrm{PN}$-level EOB coefficient $\widetilde{a}_{5}(\nu ; \ln u)$ is no more than quadratic in $\nu$, i.e.

$$
\widetilde{a}_{5}(\nu ; \ln u)=\left(a_{5}+\frac{64}{5} \ln u\right) \nu+a_{5}^{\prime} \nu^{2},
$$

without contributions of degree $\nu^{3}$ and $\nu^{4}$. We recall that similar cancellations of higher $\nu^{n}$ terms were found at lower PN orders in the EOB $A(u ; \nu)$ function. Namely, they were found to contain only terms linear in $\nu$, while $\widetilde{a}_{3}(\nu)$ could a priori have been quadratic in $\nu$, and $\widetilde{a}_{4}(\nu)$ could a priori have been cubic in $\nu$. The fact that similar remarkable cancellations still hold, according to the result of [91, at the $4 \mathrm{PN}$ level, is a clear indication that the EOB packaging of information of the dynamics in the $A(u ; n u)$ potential is quite compact. Indeed, it says that the two complicated terms $\frac{301}{1728} \nu^{3}+\frac{77}{31104} \nu^{4}$ in the energy function are already encoded in the structure of the EOB formalism. Finally, note that the full gauge-invariant content of a $4 \mathrm{PN}$ computation of the dynamics, when interpreted within the EOB formalism, is described by only three 
EOB terms: the coefficient $\widetilde{a}_{5}(\nu ; \ln u)$ in $A(u ; \nu)$, an analogous coefficient $\widetilde{\bar{d}}_{4}(\nu ; \ln u)$ in $\bar{D}(u ; \nu)$, and an additional contribution to $Q(p)$.

Regarding the waveform, let us mention another recent example where it would have been useful and clarifying to use the EOB packaging. Namely, when re-expressing it in terms of the factorized EOB waveform, the new content of the recent 3.5PN level computation by Faye, Marsat, Blanchet, and Iyer [79] of the PN-expanded quadrupolar waveform $h_{22}$, is entirely contained in an additional 3.5PN-level contribution to the supplementary phase, namely $\delta_{22}=\left(30995 / 1134 \nu+962 / 135 \nu^{2}\right) x^{7 / 2}$. Indeed, the 3.5PN-level contributions to the modulus computed in [79] were already included in the factorized EOB waveform of Ref. 67.

\section{Conclusions}

We hope that this brief review has made it clear that:

1. There is a complementarity between the various current approaches to the general relativistic two-body problem: post-Newtonian, Effective One Body, gravitational self-force and numerical relativity simulations (of both comparable-mass and extreme-mass-ratio systems).

2. The effective one body formalism offers a convenient framework for combining, in a synergetic manner, information coming from the other approaches. This formalism seems to constitute an efficient way to analytically describe the motion and radiation of circularized 10 binaries, and to provide accurate gravitational wave templates for detection and data analysis.

3. The general relativistic two-body problem is more lively than ever. It provides an example of Poincaré's sentence: "Il n'y a pas de problèmes résolus, il y a seulement des problèmes plus ou moins résolus." ["There are no (definitely) solved problems, there are only more-or-less solved problems."]

\section{References}

[1] Akcay, Sarp, Barack, Leor, Damour, Thibault and Sago, Norichika, "Gravitational self-force and the effective-one-body formalism between the innermost stable circular orbit and the light ring", Phys.Rev., D86, 104041, (2012). [DOI], arXiv:1209.0964 [gr-qc]].

[2] Baiotti, L., Damour, T., Giacomazzo, B., Nagar, A. and Rezzolla, L., "Analytic modelling of tidal effects in the relativistic inspiral of binary

${ }^{10}$ See [15] for a recent extension of the EOB formalism to non-circular (ellipticlike or hyperboliclike) motions. 
neutron stars", Phys. Rev. Lett., 105, 261101, (2010). [1009.0521 $[\mathrm{gr}-\mathrm{qc}]]$.

[3] Baiotti, L., Damour, T., Giacomazzo, B., Nagar, A. and Rezzolla, L., "Accurate numerical simulations of inspiralling binary neutron stars and their comparison with effective-one-body analytical models", Phys. Rev. $D, \mathbf{8 4}, 024017,(2011)$. 1103.3874 [gr-qc]].

[4] Baker, J.G., Centrella, J., Choi, D.I., Koppitz, M. and van Meter, J., "Gravitational wave extraction from an inspiraling configuration of merging black holes", Phys. Rev. Lett., 96, 111102, (2006). gr-qc/0511103.

[5] Barack, L., "Gravitational self force in extreme mass-ratio inspirals", Class. Quant. Grav., 26, 213001, (2009). 0908.1664 [gr-qc]].

[6] Barack, L., Damour, T. and Sago, N., "Precession effect of the gravitational self-force in a Schwarzschild spacetime and the effective one-body formalism", Phys. Rev. D, 82, 084036, (2010). [1008.0935 $[\mathrm{gr}-\mathrm{qc}]]$.

[7] Barausse, E. and Buonanno, A., "An Improved effective-one-body Hamiltonian for spinning black-hole binaries", Phys. Rev. D, 81, 084024, (2010). 0912.3517 [gr-qc]].

[8] Barausse, E. and Buonanno, A., "Extending the effective-one-body Hamiltonian of black-hole binaries to include next-to-next-to-leading spin-orbit couplings", Phys. Rev. D, 84, 104027, (2011). [1107.2904 $[\mathrm{gr}-\mathrm{qc}]]$.

[9] Barausse, Enrico, Buonanno, Alessandra, Hughes, Scott A., Khanna, Gaurav, O'Sullivan, Stephen et al., "Modeling multipolar gravitationalwave emission from small mass-ratio mergers", Phys.Rev., D85, 024046, (2012). DOI], arXiv:1110.3081 [gr-qc]].

[10] Barausse, E., Buonanno, A. and Le Tiec, A., "The complete non-spinning effective-one-body metric at linear order in the mass ratio", Phys. Rev. $D, \mathbf{8 5}, 064010,(2012)$. 1111.5610 [gr-qc]].

[11] Barausse, E., Racine, E. and Buonanno, A., "Hamiltonian of a spinning test-particle in curved spacetime. [Erratum-ibid. D 85, 069904 (2012)]", Phys. Rev. D, 80, 104025, (2009). 0907.4745 [gr-qc]].

[12] Bernuzzi, S., Nagar, A. and Zenginoglu, A., "Binary black hole coalescence in the extreme-mass-ratio limit: testing and improving the effectiveone-body multipolar waveform", Phys. Rev. D, 83, 064010, (2011). 1012.2456 [gr-qc]].

[13] Bernuzzi, S., Nagar, A. and Zenginoglu, A., "Binary black hole coalescence in the large-mass-ratio limit: the hyperboloidal layer method and waveforms at null infinity", Phys. Rev. D, 84, 084026, (2011). $[1107.5402[\mathrm{gr}-\mathrm{qc}]]$.

[14] Berti, E., Cardoso, V., Gonzalez, J.A., Sperhak, U., Hannam, M., Husa, S. and Bruegmann, B., "Inspiral, merger and ringdown of unequal mass black hole binaries: A multipolar analysis", Phys. Rev. D, 76, 064034, (2007). gr-qc/0703053.

[15] Bini, D. and Damour, T., "Gravitational radiation reaction along general orbits in the effective one-body formalism", Phys. Rev. D, 86, 124012, 
(2012). 1210.2834[gr-qc]].

[16] Bini, D., Damour, T. and Faye, G., "Effective action approach to higherorder relativistic tidal interactions in binary systems and their effective one body description", Phys. Rev. D, 85, 124034, (2012). [1202.3565 $[\mathrm{gr}-\mathrm{qc}]]$.

[17] Blanchet, L., "Second Postnewtonian Generation Of Gravitational Radiation", Phys. Rev. D, 51, 2559, (1995). gr-qc/9501030.

[18] Blanchet, L., "Gravitational-wave tails of tails, [Erratum-ibid. 22, 3381 (2005)]", Class. Quant. Grav., 15, 113, (1998). gr-qc/9710038.

[19] Blanchet, L., "Quadrupole-quadrupole gravitational waves", Class. Quant. Grav., 15, 89, (1998). gr-qc/9710037.

[20] Blanchet, L., "Gravitational radiation from post-Newtonian sources and inspiralling compact binaries", Living Rev. Rel., 5, 3, (2002). gr-qc/0202016.

[21] Blanchet, L. and Damour, T., "Radiative gravitational fields in general relativity I. General structure of the field outside the source", Phil. Trans. Roy. Soc. Lond. A, 320, 379, (1986).

[22] Blanchet, L. and Damour, T., "Postnewtonian generation of gravitational waves", Annales Poincaré Phys. Theor., 50, 377, (1989).

[23] Blanchet, L. and Damour, T., "Hereditary Effects In Gravitational Radiation", Phys. Rev. D, 46, 4304, (1992).

[24] Blanchet, L., Damour, T. and Esposito-Farèse, G., "Dimensional regularization of the third post-Newtonian dynamics of point particles in harmonic coordinates", Phys. Rev. D, 69, 124007, (2004). gr-qc/0311052.

[25] Blanchet, L., Damour, T., Esposito-Farèse, G. and Iyer, B.R., "Gravitational radiation from inspiralling compact binaries completed at the third post-Newtonian order", Phys. Rev. Lett., 93, 091101, (2004). gr-qc/0406012.

[26] Blanchet, L., Damour, T., Esposito-Farèse, G. and Iyer, B.R., "Dimensional regularization of the third post-Newtonian gravitational wave generation from two point masses", Phys. Rev. D, 71, 124004, (2005). gr-qc/0503044.

[27] Blanchet, L., Damour, T. and Iyer, B.R., "Gravitational Radiation Damping Of Compact Binary Systems To Second Postnewtonian Order, [Erratum-ibid. D 54, 1860 (1996)]", Phys. Rev. D, 51, 5360, (1995). gr-qc/9501029.

[28] Blanchet, L., Damour, T., Iyer, B.R., Will, C.M. and Wiseman, A.G., "Gravitational Radiation Damping Of Compact Binary Systems To Second Postnewtonian Order", Phys. Rev. Lett., 74, 3515, (1995). gr-qc/9501027.

[29] Blanchet, L., Detweiler, S.L., Le Tiec, A. and Whiting, B.F., "High-Order Post-Newtonian Fit of the Gravitational Self-Force for Circular Orbits in the Schwarzschild Geometry", Phys. Rev. D, 81, 084033, (2010). 1002.0726 [gr-qc]].

[30] Blanchet, L. and Faye, G., "General relativistic dynamics of compact binaries at the third post-Newtonian order", Phys. Rev. D, 63, 062005, 
(2001). gr-qc/0007051].

[31] Blanchet, L., Faye, G., Iyer, B.R. and Sinha, S., "The third postNewtonian gravitational wave polarisations and associated spherical harmonic modes for inspiralling compact binaries in quasi-circular orbits", Class. Quant. Grav., 25, 165003, (2008). [0802.1249 [gr-qc]].

[32] Blanchet, L. and Iyer, B.R., "Hadamard regularization of the third postNewtonian gravitational wave generation of two point masses", Phys. Rev. D, 71, 024004, (2005). gr-qc/0409094.

[33] Blanchet, L., Iyer, B.R. and Joguet, B., "Gravitational waves from inspiralling compact binaries: Energy flux to third post-Newtonian order, [Erratum-ibid. D 71, 129903 (2005)]", Phys. Rev. D, 65, 064005, (2002). gr-qc/0105098.

[34] Blanchet, L. and Schäfer, G., "Gravitational wave tails and binary star systems", Class. Quant. Grav., 10, 2699, (1993).

[35] Boyle, M. et al., "High-accuracy comparison of numerical relativity simulations with post-Newtonian expansions", Phys. Rev. D, 76, 124038, (2007). [0710.0158 [gr-qc]].

[36] Brézin, E., Itzykson, C. and Zinn-Justin, J., "Relativistic balmer formula including recoil effects", Phys. Rev. D, 1, 2349, (1970).

[37] Buchman, Luisa T., Pfeiffer, Harald P., Scheel, Mark A. and Szilagyi, Bela, "Simulations of non-equal mass black hole binaries with spectral methods", Phys.Rev., D86, 084033, (2012). DOI], [arXiv:1206.3015 $[\mathrm{gr}-\mathrm{qc}]]$.

[38] Buonanno, A., Chen, Y. and Damour, T., "Transition from inspiral to plunge in precessing binaries of spinning black holes", Phys. Rev. D, 74, 104005, (2006). gr-qc/0508067].

[39] Buonanno, A., Cook, G.B. and Pretorius, F., "Inspiral, merger and ringdown of equal-mass black-hole binaries", Phys. Rev. D, 75, 124018, (2007). gr-qc/0610122.

[40] Buonanno, A. and Damour, T., "Effective one-body approach to general relativistic two-body dynamics", Phys. Rev. D, 59, 084006, (1999). gr-qc/9811091.

[41] Buonanno, A. and Damour, T., "Transition from inspiral to plunge in binary black hole coalescences", Phys. Rev. D, 62, 064015, (2000). gr-qc/0001013.

[42] Buonanno, A., Pan, Y., Baker, J.G., Centrella, J., Kelly, B.J., McWilliams, S.T. and van Meter, J.R., "Toward faithful templates for non-spinning binary black holes using the effective-one-body approach", Phys. Rev. $D, \mathbf{7 6}, 104049,(2007)$. 0706.3732 [gr-qc]].

[43] Buonanno, A., Pan, Y., Pfeiffer, H.P., Scheel, M.A., Buchman, L.T. and Kidder, L.E., "Effective-one-body waveforms calibrated to numerical relativity simulations: coalescence of non-spinning, equal-mass black holes", Phys. Rev. D, 79, 124028, (2009). 0902.0790 [gr-qc]].

[44] Campanelli, M., Lousto, C.O., Marronetti, P. and Zlochower, Y., "Accurate Evolutions of Orbiting Black-Hole Binaries Without Excision", Phys. Rev. Lett., 96, 111101, (2006). gr-qc/0511048.

[45] Damour, T., "Gravitational radiation and the motion of compact bodies", 
in Deruelle, N. and Piran, T., eds., Gravitational Radiation, pp. 59-144, (North-Holland, Amsterdam, 1983).

[46] Damour, T., "The problem of motion in Newtonian and Einsteinian gravity", in Hawking, S.W. and Israel, W., eds., Three Hundred Years of Gravitation, pp. 128-198, (Cambridge University Press, Cambridge, 1987).

[47] Damour, T., "Coalescence of two spinning black holes: An effective onebody approach", Phys. Rev. D, 64, 124013, (2001). gr-qc/0103018.

[48] Damour, T., "Gravitational Self Force in a Schwarzschild Background and the Effective One Body Formalism", Phys. Rev. D, 81, 024017, (2010). 0910.5533 [gr-qc]].

[49] Damour, T., "(unpublished); cited in Ref. 6], which quoted and used some combinations of the logarithmic contributions to $a(u)$ and $\bar{d}(u) ",(2010)$.

[50] Damour, Thibault and Esposito-Farese, Gilles, "Tensor multiscalar theories of gravitation", Class.Quant.Grav., 9, 2093-2176, (1992). DOI.

[51] Damour, Thibault and Esposito-Farese, Gilles, "Testing gravity to second postNewtonian order: A Field theory approach", Phys.Rev., D53, 55415578, (1996). [DOI], arXiv:gr-qc/9506063 [gr-qc].

[52] Damour, Thibault and Esposito-Farese, Gilles, "Gravitational wave versus binary - pulsar tests of strong field gravity", Phys.Rev., D58, 042001, (1998). [DOI], arXiv:gr-qc/9803031 [gr-qc]].

[53] Damour, T. and Gopakumar, A., "Gravitational recoil during binary black hole coalescence using the effective one body approach", Phys. Rev. D, 73, 124006, (2006). gr-qc/0602117.

[54] Damour, T., Gourgoulhon, E. and Grandclément, P., "Circular orbits of corotating binary black holes: Comparison between analytical and numerical results", Phys. Rev. D, 66, 024007, (2002). gr-qc/0204011.

[55] Damour, T. and Iyer, B.R., "Multipole analysis for electromagnetism and linearized gravity with irreducible cartesian tensors", Phys. Rev. D, 43, 3259, (1991).

[56] Damour, T. and Iyer, B.R., "PostNewtonian generation of gravitational waves. 2. The Spin moments", Annales Poincaré Phys. Theor., 54, 115, (1991).

[57] Damour, T., Iyer, B.R. and Nagar, A., "Improved resummation of postNewtonian multipolar waveforms from circularized compact binaries", Phys. Rev. D, 79, 064004, (2009). 0811.2069 [gr-qc]].

[58] Damour, T., Iyer, B.R. and Sathyaprakash, B.S., "Improved filters for gravitational waves from inspiralling compact binaries", Phys. Rev. D, 57, 885, (1998). gr-qc/9708034.

[59] Damour, T., Jaranowski, P. and Schaäfer, G., "Poincaré invariance in the ADM Hamiltonian approach to the general relativistic two-body problem, [Erratum-ibid. D 63, 029903 (2001)]", Phys. Rev. D, 62, 021501, (2000). gr-qc/0003051.

[60] Damour, T., Jaranowski, P. and Schäfer, G., "Dynamical invariants for general relativistic two-body systems at the third postNewtonian approximation", Phys. Rev. D, 62, 044024, (2000). gr-qc/9912092. 
[61] Damour, T., Jaranowski, P. and Schäfer, G., "On the determination of the last stable orbit for circular general relativistic binaries at the third post-Newtonian approximation", Phys. Rev. D, 62, 084011, (2000). gr-qc/0005034.

[62] Damour, T., Jaranowski, P. and Schäfer, G., "Dimensional regularization of the gravitational interaction of point masses", Phys. Lett. B, 513, 147-155, (2001). gr-qc/0105038.

[63] Damour, T., Jaranowski, P. and Schäfer, G., "Effective one body approach to the dynamics of two spinning black holes with next-to-leading order spin-orbit coupling", Phys. Rev. D, 78, 024009, (2008). [0803.0915 $[\mathrm{gr}-\mathrm{qc}]]$.

[64] Damour, T. and Nagar, A., "Faithful Effective-One-Body waveforms of small-mass-ratio coalescing black-hole binaries", Phys. Rev. D, 76, 064028, (2007). [0705.2519 [gr-qc]].

[65] Damour, T. and Nagar, A., "Final spin of a coalescing black-hole binary: an Effective-One-Body approach", Phys. Rev. D, 76, 044003, (2007). [0704.3550 [gr-qc]].

[66] Damour, T. and Nagar, A., "Comparing Effective-One-Body gravitational waveforms to accurate numerical data", Phys. Rev. D, 77, 024043, (2008). 0711.2628 [gr-qc]].

[67] Damour, T. and Nagar, A., "An improved analytical description of inspiralling and coalescing black-hole binaries", Phys. Rev. D, 79, 081503, (2009). 0902.0136 [gr-qc].

[68] Damour, T. and Nagar, A., "Effective One Body description of tidal effects in inspiralling compact binaries", Phys. Rev. D, 81, 084016, (2010). [0911.5041[gr-qc]].

[69] Damour, T. and Nagar, A., "The Effective One Body description of the Two-Body problem", Fundam. Theor. Phys., 162, 211, (2011). 0906.1769 [gr-qc]].

[70] Damour, T., Nagar, A. and Bernuzzi, S., "to be submitted for publication", (2012).

[71] Damour, T., Nagar, A., Hannam, M., Husa, S. and Bruegmann, B., "Accurate Effective-One-Body waveforms of inspiralling and coalescing black-hole binaries", Phys. Rev. D, 78, 044039, (2008). [0803.3162 $[\mathrm{gr}-\mathrm{qc}]]$.

[72] Damour, T., Nagar, A., Nils Dorband, E., Pollney, D. and Rezzolla, L., "Faithful Effective-One-Body waveforms of equal-mass coalescing blackhole binaries", Phys. Rev. D, 77, 084017, (2008). [0712.3003 [gr-qc]].

[73] Damour, T., Nagar, A., Pollney, D. and Reisswig, C., "Energy versus Angular Momentum in Black Hole Binaries", Phys. Rev. Lett., 108, 131101, (2012). [1110.2938 [gr-qc]].

[74] Damour, T., Nagar, A. and Tartaglia, A., "Binary black hole merger in the extreme mass ratio limit", Class. Quant. Grav., 24, S109, (2007). gr-qc/0612096.

[75] Damour, T., Nagar, A. and Villain, L., "Measurability of the tidal polarizability of neutron stars in late-inspiral gravitational-wave signals", Phys. Rev. D, 85, 123007, (2012). 1203.4352 [gr-qc]]. 
[76] Damour, T. and Schäfer, G., "Higher order relativistic periastron advances and binary pulsars", Nuovo Cim. B, 101, 127, (1988).

[77] Davis, M., Ruffini, R. and Tiomno, J., "Pulses of gravitational radiation of a particle falling radially into a Schwarzschild black hole", Phys. Rev. $D, \mathbf{5}, 2932,(1972)$.

[78] Epstein, R. and Wagoner, R.V., "Post-Newtonian generation of gravitational waves", Astrophys. J., 197, 717-723, (1975).

[79] Faye, G., Marsat, S., Blanchet, L. and Iyer, B.R., "The third and a half post-Newtonian gravitational wave quadrupole mode for quasi-circular inspiralling compact binaries", Class. Quant. Grav., 29, 175004, (2012). $[1204.1043[\mathrm{gr}-\mathrm{qc}]]$.

[80] Foffa, S. and Sturani, R., "Effective field theory calculation of conservative binary dynamics at third post-Newtonian order", Phys. Rev. D, 84, 044031, (2011). 1104.1122 [gr-qc]].

[81] Foffa, S. and Sturani, R., "The dynamics of the gravitational two-body problem in the post-Newtonian approximation at quadratic order in the Newton's constant", (2012). [1206.7087 [gr-qc]].

[82] Fujita, R., "Gravitational radiation for extreme mass ratio inspirals to the 14th post-Newtonian order", Prog. Theor. Phys., 127, 583, (2012). [1104.5615 [gr-qc]].

[83] Fujita, R. and Iyer, B.R., "Spherical harmonic modes of 5.5 postNewtonian gravitational wave polarisations and associated factorised resummed waveforms for a particle in circular orbit around a Schwarzschild black hole", Phys. Rev. D, 82, 044051, (2010). [1005.2266 $[\mathrm{gr}-\mathrm{qc}]]$.

[84] Goldberger, Walter D. and Ross, Andreas, "Gravitational radiative corrections from effective field theory", Phys.Rev., D81, 124015, (2010). [DOI], arXiv:0912.4254 [gr-qc]].

[85] Goldberger, Walter D. and Rothstein, Ira Z., "An Effective field theory of gravity for extended objects", Phys.Rev., D73, 104029, (2006). [DOI], arXiv:hep-th/0409156 [hep-th]].

[86] Gonzalez, J.A., Sperhake, U., Bruegmann, B., Hannam, M. and Husa, S., "Total recoil: the maximum kick from nonspinning black-hole binary inspiral", Phys. Rev. Lett., 98, 091101, (2007). gr-qc/0610154.

[87] Hannam, M., Husa, S., Bruegmann, B. and Gopakumar, A., "Comparison between numerical-relativity and post-Newtonian waveforms from spinning binaries: the orbital hang-up case", Phys. Rev. D, 78, 104007, (2008). 0712.3787 [gr-qc]].

[88] Hannam, M., Husa, S., Sperhake, U., Bruegmann, B. and Gonzalez, J.A., "Where post-Newtonian and numerical-relativity waveforms meet", Phys. Rev. D, 77, 044020, (2008). 0706.1305 [gr-qc]].

[89] Itoh, Y. and Futamase, T., "New derivation of a third post-Newtonian equation of motion for relativistic compact binaries without ambiguity", Phys. Rev. D, 68, 121501, (2003). gr-qc/0310028.

[90] Jaranowski, P. and Schäfer, G., "3rd post-Newtonian higher order Hamilton dynamics for two-body point-mass systems ([Erratum-ibid. D 63, 029902 (2001)])", Phys. Rev. D, 57, 72-74, (1998). gr-qc/9712075. 
[91] Jaranowski, P. and Schäfer, G., "Towards the 4th post-Newtonian Hamiltonian for two-point-mass systems", Phys. Rev. D, 86, 061503, (2012). 1207.5448 [gr-qc]].

[92] Kidder, L.E., "Using Full Information When Computing Modes of PostNewtonian Waveforms From Inspiralling Compact Binaries in Circular Orbit", Phys. Rev. D, 77, 044016, (2008). 0710.0614 [gr-qc]].

[93] Konigsdorffer, C., Faye, G. and Schäfer, G., "The binary black-hole dynamics at the third-and-a-half post-Newtonian order in the ADMformalism", Phys. Rev. D, 68, 044004, (2003). gr-qc/0305048.

[94] Le Tiec, A., Barausse, E. and Buonanno, A., "Gravitational Self-Force Correction to the Binding Energy of Compact Binary Systems", Phys. Rev. Lett., 108, 131103, (2012). [1111.5609 [gr-qc]].

[95] Le Tiec, A., Blanchet, L. and Whiting, B.F., "The First Law of Binary Black Hole Mechanics in General Relativity and Post-Newtonian Theory", Phys. Rev. D, 85, 064039, (2012). 1111.5378 [gr-qc]].

[96] Le Tiec, A., Mroue, A.H., Barack, L., Buonanno, A., Pfeiffer, H.P., Sago, N. and Taracchini, A., "Periastron Advance in Black Hole Binaries", Phys. Rev. Lett., 107, 141101, (2011). 1106.3278 [gr-qc]].

[97] MacDonald, Ilana, Mroue, Abdul H., Pfeiffer, Harald P., Boyle, Michael, Kidder, Lawrence E. et al., "Suitability of hybrid gravitational waveforms for unequal-mass binaries", (2012). [arXiv:1210.3007 $[\mathrm{gr}-\mathrm{qc}]]$.

[98] Nagar, A., "Effective one body Hamiltonian of two spinning black-holes with next-to-next-to-leading order spin-orbit coupling", Phys. Rev. D, 84, 084028, (2011). 1106.4349 [gr-qc]].

[99] Nissanke, S. and Blanchet, L., "Gravitational radiation reaction in the equations of motion of compact binaries to 3.5 post-Newtonian order", Class. Quant. Grav., 22, 1007, (2005). gr-qc/0412018.

[100] Pan, Y., Buonanno, A., Boyle, M., Buchman, L.T., Kidder, L.E., Pfeiffer, H.P. and Scheel, M.A., "Inspiral-merger-ringdown multipolar waveforms of nonspinning black-hole binaries using the effective-onebody formalism", Phys. Rev. D, 84, 124052, (2011). [1106.1021 [gr-qc]].

[101] Pan, Y., Buonanno, A., Buchman, L.T., Chu, T., Kidder, L.E., Pfeiffer, H.P. and Scheel, M.A., "Effective-one-body waveforms calibrated to numerical relativity simulations: coalescence of non-precessing, spinning, equal-mass black holes", Phys. Rev. D, 81, 084041, (2010). $0912.3466[\mathrm{gr}-\mathrm{qc}]$.

[102] Pan, Y., Buonanno, A., Fujita, R., Racine, E. and Tagoshi, H., "PostNewtonian factorized multipolar waveforms for spinning, non-precessing black-hole binaries", Phys. Rev. D, 83, 064003, (2011). [1006.0431 $[\mathrm{gr}-\mathrm{qc}]]$.

[103] Pan, Y. et al., "A data-analysis driven comparison of analytic and numerical coalescing binary waveforms: Nonspinning case", Phys. Rev. $D, \mathbf{7 7}, 024014,(2008)$. 0704.1964 [gr-qc]].

[104] Pati, M.E. and Will, C.M., "Post-Newtonian gravitational radiation and equations of motion via direct integration of the relaxed Einstein equations. I: Foundations", Phys. Rev. D, 62, 124015, (2000). 
gr-qc/0007087.

[105] Pati, M.E. and Will, C.M., "Post-Newtonian gravitational radiation and equations of motion via direct integration of the relaxed Einstein equations. II: Two-body equations of motion to second post-Newtonian order, and radiation-reaction to 3.5 post-Newton", Phys. Rev. D, 65, 104008, (2002). gr-qc/0201001.

[106] Poisson, E., "Gravitational radiation from a particle in circular orbit around a black hole. I. Analytic results for the nonrotating case", Phys. Rev. D, 47, 1497-1510, (1993).

[107] Pretorius, F., "Evolution of Binary Black Hole Spacetimes", Phys. Rev. Lett., 95, 121101, (2005). gr-qc/0507014.

[108] Pretorius, F., "Binary Black Hole Coalescence", in Colpi, M. et al., ed., Relativistic Objects in Compact Binaries: From Birth to Coalescense. Springer Verlags, Canopus Publishing Limited, (2007). arXiv:0710.1338 [gr-qc]].

[109] Price, R.H. and Pullin, J., "Colliding black holes: The Close limit", Phys. Rev. Lett., 72, 3297, (1994). gr-qc/9402039.

[110] Sasaki, M., "Post-Newtonian Expansion of the Ingoing-Wave ReggeWheeler Function", Prog. Theor. Phys, 92, 17-36, (1994).

[111] Sasaki, M. and Tagoshi, H., "Analytic black hole perturbation approach to gravitational radiation", Living Rev. Rel., 6, 6, (2003). gr-qc/0306120.

[112] Scheel, M.A., Boyle, M., Chu, T., Kidder, L.E., Matthews, K.D. and Pfeiffer, H.P., "High-accuracy waveforms for binary black hole inspiral, merger, and ringdown", Phys. Rev. D, 79, 024003, (2009). [0810.1767 $[\mathrm{gr}-\mathrm{qc}]]$.

[113] Tagoshi, H. and Sasaki, M., "Post-Newtonian Expansion of Gravitational Waves from a Particle in Circular Orbit around a Schwarzschild Black Hole", Prog. Theor. Phys, 92, 745-771, (1994). gr-qc/9405062.

[114] Tanaka, T., Tagoshi, H. and Sasaki, M., "Gravitational Waves by a Particle in Circular Orbit around a Schwarzschild Black Hole", Prog. Theor. Phys, 96, 1087-1101, (1996). gr-qc/9701050].

[115] Taracchini, A., Pan, Y., Buonanno, A., Barausse, E., Boyle, M., Chu, T., Lovelace, G. and Pfeiffer, H.P. et al., "Prototype effective-one-body model for nonprecessing spinning inspiral-merger-ringdown waveforms", Phys. Rev. D, 86, 024011, (2012). [1202.0790 [gr-qc]].

[116] Thorne, K.S., "Multipole expansions of gravitational radiation", Rev. Mod. Phys., 52, 299-340, (1980).

[117] Wagoner, R.V. and Will, C.M., "Post-Newtonian gravitational radiation from orbiting point masses", Astrophys. J., 210, 764-775, (1976).

[118] Will, C.M., "Generation of post-Newtonian gravitational radiation via direct integration of the relaxed Einstein equations", Prog. Theor. Phys. Suppl., 136, 158, (1999). gr-qc/9910057.

[119] Will, C.M. and Wiseman, A.G., "Gravitational radiation from compact binary systems: gravitational waveforms and energy loss to second postNewtonian order", Phys. Rev. D, 54, 4813, (1996). gr-qc/9608012.

[120] Wiseman, A.G., "Coalescing Binary Systems Of Compact Objects To (Post)Newtonian5/2 Order.4v: The Gravitational Wave Tail", Phys. 
Rev. D, 48, 4757, (1993).

[121] Yunes, N., Buonanno, A., Hughes, S.A., Coleman Miller, M. and Pan, Y., "Modeling Extreme Mass Ratio Inspirals within the Effective-One-Body Approach", Phys. Rev. Lett., 104, 091102, (2010). 0909.4263 [gr-qc]].

[122] Yunes, N., Buonanno, A., Hughes, Pan, Y., Barausse, E., Miller, M.C. and Throwe, W., "Extreme Mass-Ratio Inspirals in the Effective-One-Body Approach: Quasi-Circular, Equatorial Orbits around a Spinning Black Hole", Phys. Rev. D, 83, 044044, (2011). [1009.6013 [gr-qc]]. 